\title{
MULTICONFIGURATION APPROACH IN THE STUDY OF ATOMIC SPECTRA
}

\author{
P. Bogdanovich \\ Vilnius University Research Institute of Theoretical Physics and Astronomy, A. Goštauto 12, LT-01108 Vilnius, Lithuania \\ E-mail: pavlas@itpa.lt \\ Received 25 February 2004
}

Dedicated to the 100th anniversary of Professor A. Jucys

\begin{abstract}
The methods to account for the correlation effects in theoretical studies of the atomic spectra by the superposition of configurations, that were developed at the Department of Theory of Atom in the Institute of Theoretical Physics and Astronomy during 50 years since the creation of multiconfiguration equations, are briefly discussed.
\end{abstract}

Keywords: multiconfiguration approach, superposition of configurations, correlation corrections, radial orbital, energy spectra

PACS: $31.10 .+\mathrm{z}, 31.25 .-\mathrm{v}$

\section{Introduction}

Multiconfiguration approximation nowadays is one of the main and most developed methods of investigation of atoms and ions. One of the basic stages in establishing this method was the work by A. Jucys [1], where the multiconfiguration Hartree-Fock equations were correctly and consistently derived for the first time. Taking into account this consideration and the following tradition of many years, in this paper these equations will be referred to as the Hartree-FockJucys (HFJ) equations. The publication of the paper [1] is closely related to the fact that A. Jucys, still being a young scientist before the World War II, has been trained by R.D. Hartree, and in the beginning of fifties - by V. Fock. Right after the above-mentioned publication, a whole series of works were performed where these equations for particular atoms were solved both numerically and analytically [2-7]. This enabled determination of the main properties of the solutions of HFJ equations and formulation of simpler to solve and easier to perceive simplified (two-configuration) equations. In all the above-mentioned publications, as well as in many others, the equations were solved "by hand", i. e. using only mechanical calculating machines. By the initiative of Prof. A. Jucys, the first electronic computer in Lithuania (BESM-2M) was obtained and a Computation Centre was established. This allowed development of a program for solving the or- dinary Hartree-Fock (HF) equations numerically [8], i. e. considerable automation of calculations of the radial orbitals. Later on, such programs were developed in series for more advanced computers as well, first in the machine codes [9] and then in the FORTRAN algorithmic language [10]. The development of one's own programs was justified mostly by the significant differences between the locally available computers and those from the West both in the structure and in the peculiarities of the algorithmic languages. The programs for numerical solution of the HF equations, developed at the Department of Theory of Atom (previously the Department of Quantum-Mechanical Computations), and many other programs, once were widely accepted in the scientific centres of the former Soviet Union. This was enhanced by the publication of 20 issues of "Collection of Programs for the Automation of Atomic Calculations" in our Institute from 1977 to 1988, where 48 FORTRAN programs were published. It must be noted that it was a personal initiative of Prof. A. Jucys to use FORTRAN for program development as early as in the beginning of the seventies of the previous century. This fact is now undoubted. It is the language that the overwhelming majority of the programs used for the atomic calculations all over the world are written in.

The author of this paper developed the program for numerical solution of the simplified HFJ equations in the course of his thesis. It was communicated at the 
All-Union seminars on the theory of atom and atomic spectra in 1971 [11] and 1972 [12]. The second seminar was held at the Institute of Spectroscopy (ISAN) near Moscow, and the members of the seminar lived in Moscow city and went to and from ISAN by a special bus. Once in the evening on the trip back to Moscow, Prof. A. Jucys seated me beside him and we had a lengthy discussion on the problems of solving the multiconfiguration equations. Then, making reference to the known properties of the solutions of this type of equations, Professor expressed a wish that it would be better not to solve the equations but rather to obtain the radial orbitals (ROs) of the virtually excited electrons directly from the Hartree-Fock functions of the shells being corrected. Regrettably, this idea has been implemented for the first time considerably later [13]. The survey of its development is one of the tasks of this work.

In the next section, the complete and simplified HFJ equations are briefly examined, as well as the main properties of their solutions. The topics of obtaining the transformed radial orbitals simulating the solutions of the multiconfiguration equations are discussed in Section 3. In Section 4, some ideas in practical application of the multiconfiguration method that have been implemented during the last decade are treated. In the majority of publications, the approximation when the interconfiguration matrix elements (ICMEs) of the Hamiltonian are taken into account is called the configuration interaction. Yet, Prof. A. Jucys has been emphatically against using this term, because there are no real physical interactions behind the nonzero values of ICMEs, but just the fact that the initial singleconfiguration approximation is not sufficiently accurate. Following the mandates of his teacher, the author in this paper uses the term of the superposition of configurations (SC), which reflects the essence of the mentioned method quite adequately. A couple of examples of the results of its application are given in Section 5.

\section{Multiconfiguration HFJ equations and some properties of their solutions}

The use of approximation where the many-electron wave function of the whole atomic configuration is built from the wave functions of individual electrons has led to the creation of the Hartree-Fock equations [14]. Application of the solutions of these equations allows to ensure the energy minimum of a particular configuration, treated in a single-configuration approximation. Such an approximation allowed in many cases obtaining a reasonable qualitative explanation of the properties of many atoms and their spectral characteristics, but a quantitative correspondence to the results of experiments remained only very rough. Meanwhile it was clear from the beginning that the singleconfiguration wave functions of the Hartree-Fock approximation are not the eigenfunctions of the Hamiltonian, i. e. the ICMEs of the kinetic energy operator and the operators of energies of interaction with the nucleus and between the electrons remain nonzero. In order to obtain sufficiently accurate energy values and the corresponding eigenfunctions, it was necessary to diagonalize the energy matrix. Application of solutions of the conventional Hartree-Fock equations for building this matrix revealed that in order to obtain high accuracy it is necessary to include a very large number of configurations that take into account even the excitation of electrons into the continuous spectra. Thus, the use of the Hartree-Fock ROs leads to a very slow convergence of the method. In order to ensure its fast convergence, it is necessary to take SC into account already in determining the ROs.

In [1], an approach has been proposed, which can be described schematically as follows. Similarly as in the case of the conventional HF equations, a Hamiltonian that includes only the nonrelativistic terms is used. The wave function $\Psi\left(K_{0} \lambda L S \mid x\right)$ of the investigated state $\lambda L S$ of the configuration $K_{0}$ being corrected is initially represented in the form of an expansion into the wave functions of terms $T L S$ ( $T$ is an array of all the intermediate momenta of the term, $L S$ are its final orbital and spin momenta) of the configuration $K$ :

$\Psi\left(K_{0} \lambda L S \mid x\right)=\sum_{K T} a\left(K_{0} \lambda L S, K T L S\right) \Psi(K T L S \mid x)$.

The summation includes the investigated configuration $K_{0}$ having a term $T_{0} L S$, with the energy and the wave function to be corrected, as well as a whole series of other configurations. They are obtainable from the investigated configuration by the single- or twoelectron excitations without the change of the parity of configuration. ROs obtained under this approximation for the single-electron states $n l$, that are absent in the configuration considered, as a rule, differ considerably from the conventional Hartree-Fock ROs and describe the physically nonexistent states. These excitations are usually called virtual, and the configurations obtained while using them - admixed configurations. The coefficients of expansion $a\left(K_{0} \lambda L S, K T L S\right)$ are assumed to be normalized to unity:

$$
\sum_{K T} a^{2}\left(K_{0} \lambda L S, K T L S\right)=1 .
$$


The wave function (2.1) is used in writing down the energy functional:

$$
\begin{aligned}
\widetilde{\Phi}\left(K_{0} \lambda L S\right)= & \sum_{K T, K^{\prime} T^{\prime}} a\left(K_{0} \lambda L S, K T L S\right) a\left(K^{\prime} T^{\prime} L S, K_{0} \lambda L S\right) \\
& \times\left[E\left(K T L S, K^{\prime} T^{\prime} L S\right)+\sum_{n l} \varepsilon_{n l}\left(K T L S, K^{\prime} T^{\prime} L S\right) \int \Psi(K T L S \mid x) \Psi\left(K^{\prime} T^{\prime} L S \mid x\right) \mathrm{d} x\right] .
\end{aligned}
$$

Here $E\left(K T L S, K^{\prime} T^{\prime} L S\right)$ are the matrix elements of the energy operator, and the summation over $n l$, including the Lagrange multipliers $\varepsilon_{n l}$, is meant to ensure the orthonormality of the solutions obtained. Variation of the expression (2.3) against the expansion coefficients, when Eq. (2.2) is taken into account, leads to the system of equations

$$
\sum_{K T} a\left(K_{0} \lambda L S, K T L S\right)\left\{\widetilde{E}\left(K T L S, K^{\prime} T^{\prime} L S\right)-E \delta\left(K T L S, K^{\prime} T^{\prime} L S\right)\right\}=0 .
$$

$\widetilde{E}\left(K T L S, K^{\prime} T^{\prime} L S\right)$ denotes the whole square brackets from Eq. (2.3), and $E$ is the eigenvalue. The system (2.4) corresponds to the secular equation for the energy matrix. Variation of Eq. (2.2) against the single-electron wave functions $\varphi_{K T L S, n l}$ leads to the system of equations of the following form:

$$
\frac{\partial \widetilde{E}(K T L S, K T L S)}{\partial \varphi_{K T L S, n l}}+2 \sum_{K^{\prime} T^{\prime} \neq K T} \frac{a\left(K_{0} \lambda L S, K^{\prime} T^{\prime} L S\right)}{a\left(K_{0} \lambda L S, K T L S\right)} \frac{\partial \widetilde{E}\left(K T L S, K^{\prime} T^{\prime} L S\right)}{\partial \varphi_{K T L S, n l}}=0 .
$$

It is the system of algebraic equations (2.4), together with the system of the integro-differential equations (2.5), that presents the multiconfiguration HFJ equations. As this system is obtained from the variation principle, the ROs obtained ensure the maximal possible energy corrections and the optimal speed of convergence of the method, that are obtainable for the investigated basis of admixed configurations. The problems of solving the HFJ equations with the use of contemporary computers are covered in detail in monographs $[15,16]$.

Even the first solutions of the HFJ equations [2-7], performed still without using the computer programs, have demonstrated that the ROs describing the single-electron states present in the adjusted configuration change only slightly as compared to their Hartree-Fock analogues. This allows one to use the conventional Hartree-Fock equations for the ROs of the adjusted configuration:

$$
\frac{\partial \widetilde{E}\left(K_{0} T_{0} L S, K_{0} T_{0} L S\right)}{\partial \varphi_{K_{0} T_{0} L S, n l}}=0 .
$$

Meanwhile, the ROs describing the electrons in the virtually excited states change very strongly. But even for the ROs of the virtually excited electrons a simplification of equations is possible, as has been proposed in [17]. The estimation of the values of the expansion coefficients indicates that in the overwhelming majority of cases $a\left(K_{0} \lambda L S, K_{0} T_{0} L S\right)$ is only slightly less than 1 and $a\left(K_{0} \lambda L S, K_{0} T_{0} L S\right) \gg a\left(K_{0} \lambda L S, K T L S\right) \ll 1$, if $K T L S \neq K_{0} T_{0} L S$. In that case it is possible to leave only one term in Eq. (2.5) in the sum over $K^{\prime} T^{\prime} L S$ that has $K^{\prime} T^{\prime} L S=K_{0} T_{0} L S$. As a result, one gets a two-configuration equation for the ROs of virtually excited electrons:

$$
\frac{\partial \widetilde{E}(K T L S, K T L S)}{\partial \varphi_{K T L S, n l}}+\frac{2}{a\left(K_{0} \lambda L S, K T L S\right)} \frac{\partial \widetilde{E}\left(K_{0} T_{0} L S, K T L S\right)}{\partial \varphi_{K T L S, n l}}=0 .
$$

It is a combination of the usual HF equations for ROs of the adjusted configuration (2.6) with the two-configuration equations for the virtually excited electrons (2.7) that constitutes the simplified HFJ equations. The equations of the form (2.7) may be obtained [18] not by neglecting particular terms in the more general Eq. (2.5), but by variation with respect to the ROs of virtually excited electrons, taking into account the orthonormality conditions, of the energy correction to the term energy $\Delta E\left(K_{0} T_{0} L S, K T L S\right)$, written in the second order of the perturbation theory as

$$
\Delta E\left(K_{0} T_{0} L S, K T L S\right)=\frac{E^{2}\left(K_{0} T_{0} L S, K T L S\right)}{E\left(K_{0} T_{0} L S, K_{0} T_{0} L S\right)-E(K T L S, K T L S)} .
$$


Equation (2.7) can be written in the explicit form:

$$
\begin{aligned}
& {\left[\frac{\mathrm{d}^{2}}{\mathrm{~d} r^{2}}-\frac{l(l-1)}{r^{2}}-U(n l \mid r)-\varepsilon_{n l, n l}\right] P(n l \mid r)} \\
& -X(n l \mid r)-\frac{2}{a\left(K_{0} \lambda L S, K T L S\right)} \Omega\left(K_{0}, K n l \mid r\right) \\
& \quad=0 .
\end{aligned}
$$

In the given integro-differential equation $U(n l \mid r)$ is the direct part of the potential and $X(n l \mid r)$ is its exchange part (including all orthogonalization conditions), and they both coincide with the corresponding potentials of the conventional Hartree-Fock method. The configurational potential $\Omega\left(K_{0}, K n l \mid r\right)$ is obtained at the variation of ICME and may be represented in the following form:

$$
\begin{aligned}
& \Omega\left(K_{0}, K n l \mid r\right) \\
& =\sum_{n_{0} l_{0}, k} \rho_{k}\left(K_{0} T_{0} L S, K T L S\right) \frac{1}{r} Y_{k}\left(n_{0} l_{0}, n l \mid r\right) \\
& \quad \times P\left(n_{0} l_{0} \mid r\right) .
\end{aligned}
$$

Here, $\rho_{k}\left(K_{0} T_{0} L S, K T L S\right)$ denotes the angular parts of the ICME and $Y_{k}\left(n_{0} l_{0}, n l \mid r\right)$ is the potential function appearing at the variation of the interconfiguration radial integrals of the electrostatic interaction $R^{k}$.

The program for solving just the simplified equations (2.9) was developed at our Institute in the beginning of the seventies [19,20]. Unfortunately, insufficient performance of the computers we had at that time did not allow us to perform extensive calculations, but it allowed us to reveal a number of interesting features of the solutions obtained. At the very beginning of exploiting the solutions of HFJ equations it was noticed that the pairwise excitations, i.e. when two equivalent electrons were excited into two equivalent states $n_{0} l_{0}^{2} \rightarrow n l^{2}$, played an important role [21]. There, it was always obtained that the $\varepsilon_{n l, n l}$ values (hereafter $\varepsilon_{n l, n l}$ are assumed to be positive) of the virtually excited electrons were considerably greater than the single-electron energies of the electrons being excited:

$$
\varepsilon_{n_{0} l_{0}, n_{0} l_{0}}<\varepsilon_{n l, n l} .
$$

While solving Eqs. (2.9) in the case of the singleelectron virtual excitations $n_{0} l_{0} \rightarrow n l$ it was noticed [19] that

$$
\varepsilon_{n_{0} l_{0}, n_{0} l_{0}}=\varepsilon_{n l, n l} .
$$

It is essential to note that in spite of the indicated properties of $\varepsilon_{n l, n l}$, the total energies of the admixed configurations containing the virtually excited electrons are always markedly higher than the total energies of the adjusted ones. If the energies of the virtually excited electrons are to be defined according to the energies of the admixed configurations, they appear to be quite high and often match the energies of electrons in the continuum spectrum, i. e. have the opposite sign. This discrepancy indicates that in the case of virtually excited electrons $\varepsilon_{n l, n l}$ cannot be treated as the singleelectron energies.

These properties of $\varepsilon_{n l, n l}$ for the case of twoconfiguration equations were mathematically proven in [18]. There, in the case of pairwise virtual excitation, the difference between the energies of configurations may be written as

$$
\begin{aligned}
& E(K T L S, K T L S)-E\left(K_{0} T_{0} L S, K_{0} T_{0} L S\right) \\
& =\varepsilon_{n_{0} l_{0}, n_{0} l_{0}}-\varepsilon_{n l, n l}+F^{0}\left(n_{0} l_{0}, n_{0} l_{0}\right)-F^{0}(n l, n l) \\
& \quad-\sum_{k} \frac{\left(l_{0}\left\|C^{(k)}\right\| l_{0}\right)^{2}}{\left(2 l_{0}+1\right)\left(4 l_{0}+1\right)} F^{k}\left(n_{0} l_{0}, n_{0} l_{0}\right) \\
& \quad+\sum_{k} \frac{\left(l\left\|C^{(k)}\right\| l\right)^{2}}{(2 l+1)(4 l+1)} F^{k}(n l, n l) \\
& \quad-\frac{2}{a\left(K_{0} \lambda L S, K T L S\right)} E\left(K_{0} T_{0} L S, K T L S\right) .
\end{aligned}
$$

Here $\left(l\left\|C^{(k)}\right\| l\right)$ denotes the submatrix element of the spherical function. In the given formula the integrals of kinetic energy and of the interaction with the nucleus are substituted by their expressions obtained while integrating the corresponding Eqs. (2.6) and (2.7). As a result, the simple expression given above is obtained, where only the integrals of electrostatic interaction within the active shells $F^{k}(n l, n l), \varepsilon_{n l, n l}$, ICME, and the coefficient of expansion are retained, and all the integrals describing the interactions with other shells cancel out. The equality (2.13) is satisfied while solving Eq. (2.7), at any values of the coefficient of expansion. If the integro-differential equations are solved selfconsistently with the corresponding secular equation, as required, then the coefficient $a\left(K_{0} \lambda L S, K T L S\right)$ in the second order of the perturbation theory satisfies the equality 
$a\left(K_{0} \lambda L S, K T L S\right)$

$$
=\frac{E\left(K_{0} T_{0} L S, K T L S\right)}{E\left(K_{0} T_{0} L S, K_{0} T_{0} L S\right)-E(K T L S, K T L S)} .
$$

Substituting (2.14) into (2.13) gives the expression

$$
\begin{aligned}
\varepsilon_{n l, n l} & -\varepsilon_{n_{0} l_{0}, n_{0} l_{0}} \\
= & E(K T L S, K T L S)-E\left(K_{0} T_{0} L S, K_{0} T_{0} L S\right) \\
& +F^{0}\left(n_{0} l_{0}, n_{0} l_{0}\right)-F^{0}(n l, n l) \\
& -\sum_{k} \frac{\left(l_{0}\left\|C^{(k)}\right\| l_{0}\right)^{2}}{\left(2 l_{0}+1\right)\left(4 l_{0}+1\right)} F^{k}\left(n_{0} l_{0}, n_{0} l_{0}\right) \\
& +\sum_{k} \frac{\left(l\left\|C^{(k)}\right\| l\right)^{2}}{(2 l+1)(4 l+1)} F^{k}(n l, n l) .
\end{aligned}
$$

The radial orbital of the admixed configuration, obtained in solving the HFJ equations, by nature is a superposition of all the physically existent ROs of the given symmetry, with all the possible energies, the continuous spectrum included. As a result, in all the cases, as mentioned earlier,

$$
E(K T L S)-E\left(K_{0} T_{0} L S\right) \gg 0 .
$$

ROs of the virtually excited electrons strongly overlap with the ROs of the adjusted configuration, consequently, the electrostatic interaction of a pair of such electrons between themselves is approximately equal, and they cancel each other in Eq. (2.15). As a result, taking into account (2.16), one obtains the inequality (2.11) from (2.15). In the case of nonpair excitations a similar reasoning may be applied leading to the analogous conclusions.

In the case of single-electron excitations the approximation, analogous to the one used in deriving Eq. (2.13), leads to the following simple expression:

$$
\begin{aligned}
E( & K T L S, K T L S)-E\left(K_{0} T_{0} L S, K_{0} T_{0} L S\right) \\
= & \frac{1}{2}\left(\varepsilon_{n_{0} l_{0}, n_{0} l_{0}}-\varepsilon_{n l, n l}\right) \\
& \quad-\frac{1}{a\left(K_{0} \lambda L S, K T L S\right)} E\left(K_{0} T_{0} L S, K T L S\right) .
\end{aligned}
$$

Taking here into account the expression for the mixing coefficient (2.13) immediately leads to the equality (2.12).

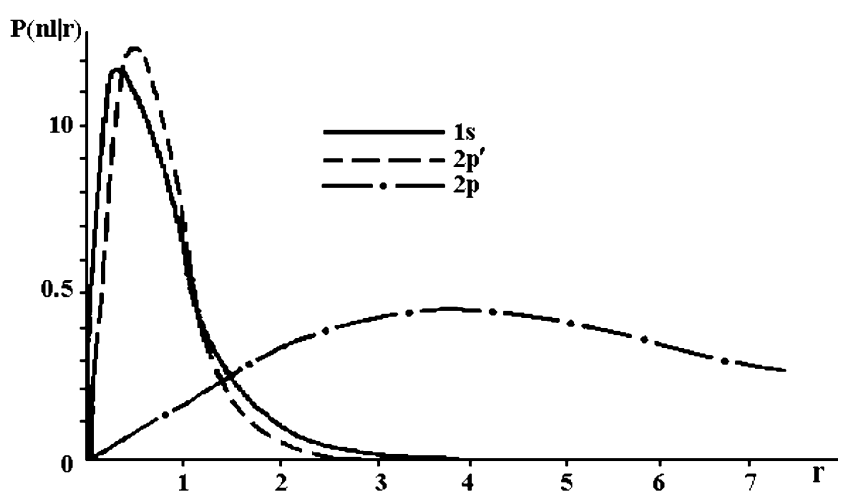

Fig. 1. Multiconfiguration RO, calculated in $\left(1 s^{2}+2 p^{2}\right) 2 p$ approximation, for $\mathrm{Li}$ I.

The behaviour of $\varepsilon_{n l, n l}$ described above is a fairly fundamental fact. The matter is that the multiconfiguration radial orbitals, similarly as the Hartree-Fock functions, decay exponentially at large distances from the nucleus:

$$
P(n l \mid r)_{r \rightarrow \infty} \sim \exp \left(-\sqrt{\varepsilon_{n l, n l}} r\right) .
$$

Therefore, the correction of wave function with the aid of ROs satisfying the conditions (2.11) or (2.12) does not change the behaviour of the wave function at large distances from the nucleus. The changes in asymptotic are possible only when solving Eqs. (2.5), when because of the influence of the configurational terms the value of the single-electron energy of the singleelectron state $n_{0} l_{0}$ being corrected is slightly changed itself.

As mentioned above, the ROs describing the virtual excitations while solving the HFJ equations change very much as compared to their Hartree-Fock analogues, by moving to the region of existence of the wave functions of electrons being corrected. An example of such behaviour of the solutions of Eq. (2.5) can be taken from [21]. Figure 1 replicates the graph from that paper. There the radial orbitals are given that are obtained while solving the multiconfiguration equations for the lithium atom in the approximation $\left(1 s^{2}+2 p^{2}\right) 2 p$. It is clearly seen in the figure how much the RO of the virtually excited electrons overlaps with $P(1 s \mid r)$, thus being thoroughly distinct from the Hartree-Fock function $P(2 p \mid r)$ of the same symmetry. While investigating the pairwise excitations such a behaviour is quite characteristic of the ROs. In that, the ROs demonstrate quite a weak dependence on the particular term of the adjusted configuration.

A totally different situation arises when the singleelectron excitations are used. This is clearly seen from the results of [19]. As an example the graphs of ROs 


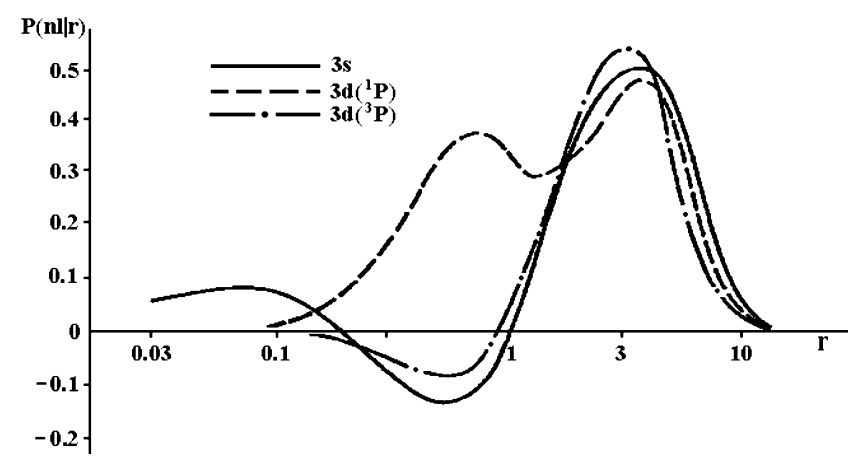

Fig. 2. Multiconfiguration RO, calculated in $2 p^{5}(3 s+3 d)$ approximation, for $\mathrm{Ne}$ I.

Table 1. Correlation corrections $\Delta E$ and expansion coefficients $a$ obtained for Ca XVII.

\begin{tabular}{lccr}
\hline & SHFC & SC & \multicolumn{1}{c}{ RSC } \\
\hline$\Delta E\left(2 s^{2}-2 p^{2}\right)$ & -0.29460 & -0.26900 & -0.2972 \\
$a\left(2 s^{2}-2 p^{2}\right)$ & 0.3041 & 0.2746 & 0.3036 \\
\hline
\end{tabular}

from that work are given in Fig. 2 that are obtained using the two-configuration approximation $2 p^{5}(3 s+3 d)$ for the $\mathrm{Ne} \mathrm{I}$ atom. As the adjusted configuration has two terms, ${ }^{1} \mathrm{P}$ and ${ }^{3} \mathrm{P}$, the RO of the virtually excited electron $3 d$ has been determined for each of the terms. It is seen from the graph that the function obtained for the case ${ }^{3} \mathrm{P}$ very closely follows the behaviour of the radial orbital of the $3 s$ electron. In the case of ${ }^{1} \mathrm{P}$ term the behaviour of $P(3 d \mid r)$ is totally different. The radial orbital has no nodes, but two "humps" appear. Thus, in the case of single-electron virtual excitations a strong dependence of solutions of the HFJ equations upon the term is detected. This is related to the peculiarities of the ICME at such excitations and is discussed in the next section.

Some questions arising in solving the HFJ equations for highly charged ions are treated in [22]. The problem lies in the fact that in such a case a considerable influence upon the difference of energies between the adjusted configuration and the admixed configurations is due to the relativistic corrections that in this case are calculated in the Breit-Pauli approximation. These may be taken into account by solving the secular equation, but are not included into the integro-differential equations. This influence gets particularly noticeable at the virtual quasi-degenerate excitation, i. e. without the change of the principal quantum number. As an example, the results obtained in the two-configuration approximation $1 s^{2}\left(2 s^{2}+2 p^{2}\right)$ for the Ca XVI ion while using three sorts of correcting ROs are presented in Table 1. The first approximation is a superposition of the Hartree-Fock configurations (SHFC), i. e. when all the ROs are the solutions of HF equations; the second and the third cases use the solutions of the multiconfiguration equations obtained without including the relativistic corrections (SC) and with including those in the secular equation (RSC). As seen from the table, in this case the solving of HF equations performed without accounting for the relativistic corrections makes the energy correction even less as compared to the superposition of the Hartree-Fock configurations, because the energy calculation is performed in the Breit-Pauli approximation. Only the account of this factor while solving the two-configuration equation allowed to obtain some increase of the energy correction, meanwhile the mixing coefficient had remained smaller than in the SHFC approximation. This example also demonstrates the known fact that while mixing the quasi-degenerate configurations the use of conventional Hartree-Fock functions is quite well founded. Physically, this is justified by the consideration that the Hartree-Fock functions corresponding to the states with the same values of $n$ usually have roughly the same average distance from the nucleus and, as a result, overlap strongly.

\section{Transformed ROs as an alternative to the solutions of HFJ equations}

Solution of the HFJ equations with thorough selfconsistency of ROs as well as of the coefficients of expansion still constitutes a quite complex computational problem. In addition, the complexity of it increases fast with the growth of the basis used. This problem was still more complex thirty years ago, when the possibilities of the computers used, both in speed and in the available memory, were some tens of thousands times lower than those of the contemporary ones. It was this factor that stimulated the idea to create a method allowing generating a set of radial orbitals for the virtually excited electrons in a simple way. There, such ROs have to imitate well the main features of the solutions of HFJ equations, which must ensure a quite fast convergence with respect to the number of configurations superposed.

As seen from the brief review presented in the previous section, the main requirement for the correcting $\mathrm{RO}$ is its strong overlap with the RO being corrected and the preservation of the asymptotic (2.18) of the Hartree-Fock function. Still one more necessary condition always observed by the solutions of Eqs. (2.5), 
(2.6), and (2.7) is their similar behaviour at the origin of coordinates:

$$
P(n l \mid r)_{r \rightarrow 0} \sim r^{l+1} .
$$

Following these considerations, it was proposed in [13] to obtain the ROs of virtually excited electrons from the Hartree-Fock functions of the adjusted configuration with the aid of a most simple transformation:

$$
\begin{aligned}
& P_{\mathrm{tr}}(n l \mid r) \\
& =N\left[r^{k} P_{\mathrm{HF}}\left(n_{0} l_{0} \mid r\right)\right. \\
& \left.\quad-\sum_{n^{\prime}<n} P\left(n^{\prime} l \mid r\right) \int_{0}^{\infty} P\left(n^{\prime} l \mid r\right) r^{k} P_{\mathrm{HF}}\left(n_{0} l_{0} \mid r\right) \mathrm{d} r\right], \\
& \quad k \geq l-l_{0}, \quad k>0 .
\end{aligned}
$$

In this case the transforming function is just a power of the radial variable, the summation over $n$ is necessary for ensuring the orthogonality of all the ROs, and the factor $N$ is used for normalization. The restrictions upon $k$ allow one to observe the condition (3.1). In the case $k=l-l_{0}>0$ this proportionality emerges immediately, and in the case $k>l-l_{0}>0$ it is guaranteed through the orthogonalization. As the RO being transformed decays exponentially at long distances (see Eq. (2.18)), this feature is preserved at any finite $k$ for the TRO with the same exponent, only this is pronounced at larger distances from the nucleus. As noted in the previous section, this corresponds to the properties of the solutions of HFJ equations in the case of single-electron excitations.

Applying the transformation (3.2) one may rather easily obtain large bases of the radial orbitals. They have been widely and quite successfully used in a whole series of publications [13,23-30]. There they have been applied both at the conventional SC and at the simplified account of the correlation effects in the second order of the perturbation theory. The simplified account of the correlation effects is most completely described in [31], and the main ideas are given at the end of the next section.

It is easy to see that the expression (3.2) may be interpreted as a result of variation with respect to $P(n l \mid r)$ of the following functional:

$$
\begin{aligned}
\widetilde{\Phi}= & \int_{0}^{\infty} P_{\mathrm{tr}}(n l \mid r) r^{k} P_{\mathrm{HF}}\left(n_{0} l_{0} \mid r\right) \mathrm{d} r \\
& +\sum_{n^{\prime}<n} \varepsilon_{n l, n^{\prime} l} \int_{0}^{\infty} P_{\mathrm{tr}}(n l \mid r) P\left(n^{\prime} l \mid r\right) \mathrm{d} r,
\end{aligned}
$$

i. e. of satisfying the requirement of maximum of the corresponding integral of the power of radial variable, together with the orthonormality of the obtained solution. While using the transformation (3.2), as well as the next ones, the values of the nondiagonal Lagrange multipliers are expressed through the values of the corresponding integrals. The maximum of the first integral in Eq. (3.3) determined the success of applying the TROs (3.2) in studying the dipole polarizability of atoms [32]. As follows from [33], the maximum of integrals of the powers of the radial variable is also a substantial factor in calculating the matrix elements of the corresponding powers of the operator of electric transition in its length form. The compliance with the requirement of maximum of Eq. (3.3) allows one, even at a comparatively small set of the admixed configurations, to obtain sufficiently reliable characteristics of the electron transitions. In the same work it is shown that the TROs (3.2) at $k=1$ are the solutions of perturbative equations for the operator of electric dipole transition written in the velocity form. This, by consequence, ensures the plausibility of the values of matrix elements calculated while using such an operator.

It is very simple to get the TROs ensuring the maximum of ICME [18]. For this purpose, it is sufficient to perform the variation of the following functional:

$$
\begin{aligned}
\widetilde{\Phi}= & \sum_{n_{0} l_{0}, k} \rho_{k}\left(K_{0} T_{0} L S, K T L S\right) \\
& \times R^{k}\left(n l, n_{0} l_{0} ; n_{0} l_{0}, n_{1} l_{1}\right) \\
& +\sum_{n^{\prime}<n} \varepsilon_{n l, n^{\prime} l} \int_{0}^{\infty} P_{\operatorname{tr}}(n l \mid r) P\left(n^{\prime} l \mid r\right) \mathrm{d} r .
\end{aligned}
$$

The first sum in Eq. (3.4) is an ICME written in the given expression for the case of the single-electron excitation $\left(n_{1} l_{1} \rightarrow n l, l_{1} \neq l\right)$. The variation of Eq. (3.4) leads to the following expression for the TRO:

$$
\begin{aligned}
& P_{\mathrm{tr}}(n l \mid r) \\
& =N\left[\sum_{n_{0} l_{0}, k} \rho_{k}\left(K_{0} T_{0} L S, K T L S\right)\right. \\
& \quad \times \frac{1}{r} Y_{k}\left(n_{0} l_{0}, n_{1} l_{1} \mid r\right) P\left(n_{0} l_{0} \mid r\right) \\
& \quad-\sum_{n^{\prime} l} P\left(n^{\prime} l \mid r\right) \sum_{n_{0} l_{0}, k} \rho_{k}\left(K_{0} T_{0} L S, K T L S\right) \\
& \left.\quad \times R_{k}\left(n^{\prime} l, n_{0} l_{0} ; n_{0} l_{0} n_{1} l_{1}\right)\right] .
\end{aligned}
$$


In the case of a two-electron virtual excitation instead of a simple transformation (3.5) it is necessary to solve the Fredholm equation of the second kind. Still, the practical application of the TROs ensuring the maximum ICME has shown that they do not improve the convergence of the configuration superposition, and even worsen it as compared to the use of the powers of the radial variable.

The use of the results of transformation (3.5) allows one to obtain quite simple explanations of the behaviour of solutions of the HFJ equations in the case of single-electron excitations. So, for the pair of configurations $2 p^{5}(3 s+3 d)$ of the neon atom, investigated in the previous section (Fig. 2), the TRO for the term ${ }^{3} \mathrm{P}$ contains a single transformation of the form (3.5),

$$
P_{\mathrm{tr}}\left(3 d^{3} \mathrm{P} \mid r\right)=N \frac{2}{\sqrt{5}} \frac{1}{r} Y_{2}(2 p, 2 p \mid r) P(3 s \mid r),
$$

and for the term ${ }^{1} \mathrm{P}$ - two ones:

$$
\begin{aligned}
P_{\mathrm{tr}}\left(3 d^{1} \mathrm{P} \mid r\right)= & N\left[\frac{2}{\sqrt{5}} \frac{1}{r} Y_{2}(2 p, 2 p \mid r) P(3 s \mid r)\right. \\
& \left.-\frac{\sqrt{2}}{3} \frac{1}{r} Y_{1}(2 p, 3 s \mid r) P(2 p \mid r)\right] .
\end{aligned}
$$

This allows one to understand at least qualitatively why the ROs of the virtually excited electrons depend on the term so strongly, and why in the case of ${ }^{3} \mathrm{P}$ they follow the course of $P(3 s \mid r)$ so well, while in the case of ${ }^{1} \mathrm{P}$ they contain two humps, the first of which corresponds to the position of the maximum of $P(2 p \mid r)$.

The TROs (3.2) along with the solutions of the HFJ equations were successfully used in [34] correcting the data on the electron densities in the nucleus, which play quite an important role in some nuclear processes. There, instead of the condition (3.1) while obtaining the TROs, a more stringent condition was used:

$$
P(n l \mid r)_{r \rightarrow 0} \sim r^{l+1}\left(1-\frac{Z}{l+1} r\right) .
$$

This made it necessary to introduce some additional restrictions upon the values of $k$ in Eq. (3.2), but practically it did not worsen the convergence of the method.

A wide comparison of the properties of the simplest TROs (3.2) to the solutions of HFJ equations was performed in [35]. As it is seen from the examples given there and from the practice of use of the simplest TROs, such an approximation is quite efficient. Meanwhile, these TROs do not reflect sufficiently the properties of the solutions of HFJ equations allowing with a single TRO to account for just about $80 \%$ of the correlation energy as compared to the corresponding multiconfiguration function. While the TROs were used mainly in order to take into account the correlations in the second order of the perturbation theory, this could not be treated as an essential drawback, because, as known, the second order of the perturbation theory gave somewhat higher estimates. In the beginning of nineties with the transition to the use of personal computers in the calculations and with a fast growth of their efficiency and the amount of memory, the direct inclusion of more and more admixed configurations in the energy matrix has become possible. This has posed still higher requirements to the quality of TROs used and has led to the development of a new class of the radial orbitals the transformed radial orbitals with a variable parameter (TROVP) [36]. The derivation of TROVP can be presented as follows:

$$
\begin{aligned}
& P_{\mathrm{tr}}(n l \mid r) \\
& =N\left[f(k, m, B \mid r) P_{\mathrm{HF}}\left(n_{0} l_{0} \mid r\right)-\sum_{n^{\prime}<n} P\left(n^{\prime} l \mid r\right)\right. \\
& \left.\quad \times \int_{0}^{\infty} P\left(n^{\prime} l \mid r\right) f(k, m, B \mid r) P_{\mathrm{HF}}\left(n_{0} l_{0} \mid r\right) \mathrm{d} r\right] .
\end{aligned}
$$

In this case a transforming function $f(k, m, B \mid r)$ containing three parameters is used. The choice of the form of this function was determined by the following considerations $[18,36]$. At the origin of coordinates it should behave as the simplest transformation from (3.2), because this allows one to get the right behaviour of the TRO near the nucleus. Meanwhile, the transforming function $r^{k}$ already at $k$ values of a few units grows very fast in the outer region of the atom, pushing the maximum of a TRO too much into the outer region. Consequently, the TRO should diminish at large $r$. Only this may allow one to obtain the TROs that reflect the properties of the real multiconfigurational ROs to the fullest. This also corresponds to the properties of the transformation (3.3) that ensures the maximum of ICME. On the basis of the said above, in [36] it has been proposed to use the transforming functions of two types, the algebraic:

$$
\begin{aligned}
& f_{a}(k, m, B \mid r)=\frac{r^{k}}{B+r^{m}}, \\
& m \geq k, \quad k \geq l-l_{0}, \quad k>0, \quad B>0
\end{aligned}
$$


and the exponential one:

$$
\begin{aligned}
& f_{e}(k, m, B)=r^{k} \exp \left(-B r^{m}\right), \\
& \quad k \leq l-l_{0}, \quad k>0, \quad m>0, \quad B>0 .
\end{aligned}
$$

The algebraic transforming function allows one to retain the convergence to zero (2.18) of the used HartreeFock RO being transformed (this corresponds to the properties of the solutions of HFJ equations in the case of single-electron excitations), and the exponential one always makes the convergence faster (this corresponds to the properties of the solutions of HFJ equations in the case of two-electron excitations). Both transforming functions considered contain two integer-valued parameters $(k$ and $m)$ and a freely variable parameter $B$.

The presence of the integer-valued parameters provides the possibility to use a vast set of most different transformations, and the parameter $B$ makes the choice infinitely large. Consequently, it is necessary to set a criterion that would constitute a basis for making the choice of both the integer-valued parameters and the parameter $B$. As such a criterion the energy correction is used, which is written in the second order of perturbation theory and is averaged over all the terms of the adjusted configuration:

$$
\Delta E\left(K_{0}, K\right)=\frac{\Theta\left(K_{0}, K\right)}{g\left(K_{0}\right)\left[\bar{E}\left(K_{0}\right)-\bar{E}(K)\right]} .
$$

Here

$$
\begin{aligned}
\Theta\left(K_{0}, K\right)= & \sum_{T_{0} T L S}(2 L+1)(2 S+1) \\
& \times E^{2}\left(K_{0} T_{0} L S, K T L S\right)
\end{aligned}
$$

represents the sum of squares of the ICMEs. The analytic expressions for (3.13) at all the possible types of virtual excitations may be found in $[37,38]$. The denominator of the expression (3.12) contains the statistical weight of the adjusted configuration

$$
g\left(K_{0}\right)=\sum_{T_{0} L S}(2 L+1)(2 S+1)
$$

and the difference of the mean configuration energies. The latter may be obtained by averaging the energy of each configuration according to the conventional formula

$$
\begin{aligned}
& \bar{E}(K)= \\
& \frac{\sum_{T L S}(2 L+1)(2 S+1) E(K T L S, K T L S)}{g(K)},
\end{aligned}
$$

which leads to the well-known simple expression. It is possible to perform a considerably more complex but also sounder in this case averaging that uses not the statistical weights of the terms (3.15), but the squares of the ICMEs:

$$
\begin{aligned}
& \bar{E}\left(K_{0}\right)-\bar{E}(K) \\
& \rightarrow \overline{E\left(K_{0} T_{0} L S\right)-E(K T L S)} \\
& =\Omega^{-1}\left(K_{0}, K\right) \sum_{T_{0} T L S}(2 L+1)(2 S+1) \\
& \quad \times E^{2}\left(K_{0} T_{0} L S, K T L S\right) \\
& \times\left[E\left(K_{0} T_{0} L S, K_{0} T_{0} L S\right)\right. \\
& \quad-E(K T L S, K T L S)]
\end{aligned}
$$

The analytical expressions for calculating the energy difference according to the formula (3.16) and the discussion of the influence of such an approach upon the averaged energy correction may be found in [39].

The paper [36] contains a large number of tables demonstrating the properties of the TROVPs. In the present publication a single table from [36] is chosen as an example, which illustrates the possibilities of the TROVPs. Table 2 contains the energy corrections obtained in the two-configuration approximation for the pair of configurations $3 d^{10}+3 d^{8} n l^{2}$ of the nickel isoelectronic series. In the table the results obtained with the aid of the solutions of HFJ equations are compared to the corrections obtained while using the TROVP. The transformations used are marked in the parentheses. First, the quantum numbers of the Hartree-Fock RO being transformed are indicated, and after the vertical dash - the type of transformation and the values of parameters $k$ and $m$ are given. As it is seen from the table, the best TROVP give the corrections almost coinciding with the results of using the solutions of HFJ equations. The analogous data were obtained also while investigating the different virtual excitations for most various ions and different ionization degrees. The TROVPs in that paper are not compared graphically to the solutions of HFJ equations because their curves on the graphs practically coincide.

The TROVPs, of course, cannot reproduce in all the cases the solutions of HFJ equations for a particular term at single-electron excitations. In the case of the example above $2 p^{5}(3 s+3 d)$ Ne I, two transformed radial orbitals are necessary, one of which is obtained from $P(3 s \mid r)$ and the second one from $P(2 p \mid r)$. The superposition of such TROVPs may reproduce the features of $P(3 d \mid r)$ that are obtained for ${ }^{3} \mathrm{P}$ and ${ }^{1} \mathrm{P}$. 
Table 2. Correlation corrections $\Delta E\left(3 d^{10}+3 d^{8} n l^{2}\right)$ for the isoelectronic series of nickel.

\begin{tabular}{ccccccc}
\hline$n l$ & $\left(n^{\prime} l^{\prime} \mid t k m\right)$ & $Z=30$ & $Z=32$ & $Z=34$ & $Z=37$ & $Z=40$ \\
\hline $4 s$ & HFJ & 0.001627 & 0.001169 & 0.000968 & 0.000815 & 0.000731 \\
& (3ple31) & $\mathbf{0 . 0 0 1 6 1 3}$ & 0.001155 & 0.000951 & 0.000795 & 0.000708 \\
& (3sle32) & 0.001609 & 0.001155 & 0.000955 & 0.000802 & 0.000717 \\
& (3sle41) & 0.001218 & $\mathbf{0 . 0 0 1 1 5 9}$ & $\mathbf{0 . 0 0 0 9 6 2}$ & $\mathbf{0 . 0 0 0 8 1 0}$ & $\mathbf{0 . 0 0 0 7 2 5}$ \\
\hline $4 p$ & MCRO & 0.012007 & 0.009570 & 0.008365 & 0.007354 & 0.006744 \\
& (3ple31) & $\mathbf{0 . 0 1 1 9 6 2}$ & 0.009521 & 0.008304 & 0.007282 & 0.006666 \\
& (3sle31) & 0.011952 & 0.009537 & 0.008330 & $\mathbf{0 . 0 0 7 3 1 2}$ & $\mathbf{0 . 0 0 6 6 9 8}$ \\
& (3sla32) & 0.011915 & $\mathbf{0 . 0 0 9 5 3 9}$ & $\mathbf{0 . 0 0 8 3 4 1}$ & 0.007287 & 0.006640 \\
& (3sle32) & 0.011887 & 0.009428 & 0.008213 & 0.007193 & 0.006576 \\
\hline \multirow{2}{*}{$4 d$} & MCRO & 0.247370 & 0.196216 & 0.169875 & 0.147532 & 0.134232 \\
& (3dla22) & $\mathbf{0 . 2 4 7 2 8 2}$ & $\mathbf{0 . 1 9 5 4 2 7}$ & $\mathbf{0 . 1 6 8 9 5 2}$ & 0.146594 & 0.133286 \\
& (3dle21) & 0.246965 & 0.195211 & 0.168801 & 0.146463 & 0.133183 \\
& (3dla33) & 0.245887 & 0.194803 & 0.168705 & $\mathbf{0 . 1 4 6 6 2 9}$ & $\mathbf{0 . 1 3 3 4 7 0}$ \\
& (3dla23) & 0.245749 & 0.194595 & 0.168472 & 0.146413 & 0.133275 \\
\hline \multirow{2}{*}{$4 f$} & MCRO & 0.166144 & 0.181441 & 0.191571 & 0.201964 & 0.208808 \\
& (3dle21) & $\mathbf{0 . 1 6 5 8 7 7}$ & $\mathbf{0 . 1 8 1 1 4 9}$ & 0.191251 & 0.201606 & $\mathbf{0 . 2 0 8 9 4 5}$ \\
& (3dla13) & 0.165865 & 0.181147 & $\mathbf{0 . 1 9 1 2 8 9}$ & $\mathbf{0 . 2 0 1 6 9 8}$ & 0.208409 \\
& (3dle12) & 0.165185 & 0.180536 & 0.190705 & 0.201139 & 0.207928 \\
& (3dla14) & 0.164967 & 0.180163 & 0.190326 & 0.200694 & 0.206510 \\
\hline & & & & & &
\end{tabular}

The properties of the TROVPs were investigated in [36], and their use in actual calculations of spectra of complex configurations [40-50] has demonstrated that the TROVPs by their properties in practice are not inferior to the solutions of multiconfiguration equations. Moreover, it appeared that both transformations after the parameter adjustment led to the same corrections. Therefore, in the latest publications usually only the exponential transformation (3.11) is used. The experience gained in calculations while using the TROVPs allowed us to almost completely automate their calculation. Only the adjusted configuration and the quantum numbers for a virtual excitation must be given in input. The program automatically selects the admixed configuration having the given virtual excitation that gives the largest averaged correction (3.12), selects the HartreeFock function to be transformed, and optimizes the values of all the parameters, too. This allows one to compile the extensive bases of the radial orbitals without any value judgement and to apply them successfully for achieving high accuracy and reliability of calculations.

\section{Topics of the optimization of basis of admixed configurations and the reduction of order of multiconfigurational matrices}

In solving the HFJ equations, as well as in using the methods described in the previous section, it is possible to compile quite extensive bases of the radial orbitals that later on are used for calculating the multiconfiguration matrix of the energy operator. However, compiling the basis of ROs is only the first stage of the calculation. Moreover, the wider the basis, the more admixed configurations are possible to create by using it. It may be taken approximately that the quantity of admixed configurations is proportional to the squared number of ROs describing the virtual excitations. Thus, the expansion of the basis that is necessary for the higher accuracy and reliability of theoretical results leads to fast growth of the number of possible admixed configurations and the related number of the terms accounted for, which in this case are often called the configuration state functions (CSFs). But the role of different admixed configurations is not nearly the same. The quite simple empirical rules exist that allow one to select a dozen of configurations that have to influence the given configuration most of all. On one hand, these rules are not of absolute character and, on the other hand, they are totally worthless when many hundreds of configurations must be selected from thousands of the possible ones.

A special method has been designed [51] for the impartial solving of this problem. It is based on calculating the sum of squares of the ICMEs $\Theta\left(K_{0}, K\right)$ (3.12) and the averaged energy distances between configurations, which are defined in the usual manner (3.15) as well as with the account of ICMEs (3.16). The presence of these quantities relating the admixed configura- 
tion to the one being corrected allows one to determine according to (3.12) the averaged energy correction, the magnitude of which may serve as a criterion in the selection. As a criterion one may also use the averaged weights of the admixed configurations that are determined according to the formula

$$
W\left(K_{0}, K\right)=\frac{\Theta\left(K_{0}, K\right)}{g\left(K_{0}\right)\left[\bar{E}\left(K_{0}\right)-\bar{E}(K)\right]^{2}} .
$$

In doing so, two definitions of the energy difference may be used in (4.1), too. Such an approach has been used in our calculations for more than a decade already, and justifies itself completely. The corresponding program is published in [52]. It allows one to evaluate and to select thousands of admixed configurations in seconds and makes the calculations substantially easier.

In spite of how refined a program of calculating and diagonalizing the energy matrix is, it depends on the possibilities of the computer used and imposes certain restrictions upon the order of matrices being calculated and diagonalized. The order of matrices themselves depends less on the number of the configurations taken into account than on the number of the CSFs present there. Usually not nearly all configuration state functions present in the admixed configuration have the ICMEs connecting them with the terms of the adjusted configuration. This, first, is caused by the known diagonality of the matrix elements of the Hamiltonian used with respect to the final orbital and spin angular momenta $L S$. In the case of two-electron virtual excitations (and these are more ample than single-electron ones) still other selection rules may be valid.

Let us treat the adjusted configuration $2 s^{2} 2 p^{4} 3 d$ and the admixed one $2 s 2 p^{3} 3 d 4 d 4 f$ (hereinafter the passive occupied inner shells are omitted in the designation of a configuration). A set of terms of the adjusted configuration is written as follows:

$$
2 s^{2}{ }^{1} \mathrm{~S} 2 p^{4} L_{20} S_{20} 3 d^{2} \mathrm{D} L_{0} S_{0} .
$$

The given admixed configuration has 2065 terms in total, that can be symbolically described as

$$
\begin{gathered}
2 s^{2} \mathrm{~S} 2 p^{3} L_{2} S_{2}\left(L_{12} S_{12}\right) 3 d^{2} \mathrm{D}\left(L_{123} S_{123}\right) \\
4 d^{2} \mathrm{D}\left(L_{1234} S_{1234}\right) 4 f^{2} \mathrm{~F} L S .
\end{gathered}
$$

In (4.2) as well as in (4.3) the sequential coupling of momenta as determined in the complex of programs [53] is used. If of the whole set (4.3) only the terms having final momenta coinciding with the final momenta of the adjusted configuration are kept, i. e. the $\delta\left(L_{0} S_{0}, L S\right)$ property of ICMEs of the Hamiltonian is taken advantage of, the number of terms (4.2) is reduced to 1054 .

Further selection of CSFs of the admixed configuration at the sequential coupling of momenta and the given traditional position of shells is impossible. Now let us move the virtually excited electrons to the beginning of the configuration:

$$
\begin{gathered}
4 d^{2} \mathrm{D} 4 f^{2} \mathrm{~F}\left(L_{12}^{\prime} S_{12}^{\prime}\right) 2 s^{2} \mathrm{~S}\left(L_{123}^{\prime} S_{123}^{\prime}\right) \\
2 p^{3} L_{4} S_{4}\left(L_{1234}^{\prime} S_{1234}^{\prime}\right) 3 d^{2} \mathrm{D} L_{0} S_{0} .
\end{gathered}
$$

The energy operator while calculating the ICMEs in this case acts upon the "edge" electron $3 d$ neither in the adjusted configuration nor in the admixed one. This provides the possibility to introduce an additional restriction clause: $\delta\left(L_{20} S_{20}, L_{1234}^{\prime} S_{1234}^{\prime}\right)$. As a result, the number of terms of the admixed configuration is reduced to 187 .

Still, these are not all possible restrictions. Each ICME connecting the given admixed configuration with the one being corrected contains two-configuration matrix elements $\left\langle 2 s 2 p L^{\prime \prime} S^{\prime \prime}|h| 4 d 4 f L^{\prime \prime} S^{\prime \prime}\right\rangle$ as factors, and they also have the properties of diagonality with respect to the final orbital and spin angular momenta. As the momenta of virtually excited electrons in (4.4) are the coupled ones, it is possible to require that $L_{12}^{\prime} S_{12}^{\prime}$ be only such that are possible for the pair of electrons $2 s 2 p$, i. e. ${ }^{1} \mathrm{P}$ or ${ }^{3} \mathrm{P}$. This reduces the number of CSFs to be accounted for while calculating the matrix to 58 . Thus, after switching to a new layout of shells we succeeded in lowering the number of CSFs by more than 35 times. Not a single ICME was lost in that. Just the number of nonzero ICMEs while using the coupling (4.4) was reduced considerably, and their modules became considerably larger, because the sum of squares of ICMEs remained the same.

Such an approach to the selection of CSFs is described in detail in [54]. Hereafter such basis is defined as refined, in contrast to the basis obtained without transposing the quantum numbers of virtually excited electrons, which is defined by the term "traditional". The refined basis provides the reduction of the number of CSFs used up to several tens of times. The program of generating the terms used for this is published in [55]. The considerable reduction of the set of CSFs allows one to increase the number of the admixed configurations many times, which induces higher accuracy of calculations. It follows from the said above that the number of CSFs often given in papers as the main criterion of the complexity of calculations is not unam- 
biguous enough. Physically, a lot more important is the number of virtual excitations used, i. e. the number of admixed configurations.

An important role while calculating the energy spectra is played by the program that forms and diagonalizes the energy operator matrix. There, differently from calculating the ROs, the Hamiltonian includes not just the electrostatic electron interaction, but also the magnetic interactions such as "spin-orbit" and others. This leads to the situation that the energy matrix becomes nondiagonal with respect to the final angular momenta $L S$ and retains the diagonality only with respect to the total angular momenta $J$ of particular states. Only a small part adjusted corresponding to the energy levels of the adjusted configuration of all the eigenvalues of the energy operator matrix is physically relevant. This fact has led to the establishment of a method of partial diagonalization [56] that allows for comparatively fast obtaining of the eigenvalues in question. Its further development has led to the introduction of a method of sequential diagonalization, where first the necessary eigenvalues and then the corresponding eigenfunctions are determined. This allows one to diagonalize the symmetric matrices with the minimal use of hard disk drives in the case when the triangular part of the matrix fits into the RAM of a computer. So, while using a computer with $0.5 \mathrm{~GB}$ of RAM, our program allows us to diagonalize the matrices of the order of over ten thousand.

Further refinement of programs has led to creation of a method of separate diagonalization. As mentioned earlier, the "spin-orbit" interaction and other relativistic corrections taken into account in the Breit-Pauli approximation lead to the nondiagonality of the energy matrix with respect to the final angular momenta $L S$. But the corrections due to the operators having $J$-dependent matrix elements usually are comparatively small and can be neglected while calculating the matrix elements for the majority of the admixed configurations. Such an approximation allows one to perform the diagonalization of the matrix for particular pairs of final momenta first, and only then, by using the eigenvalues and eigenfunctions obtained, to take into account the $J$-dependent relativistic corrections for only those configurations that are close in energy [54]. While doing so, the order of the matrix for $J$ is reduced hundreds of times practically without loss of accuracy. As every value of $J$ is made of several possible pairs $L S$ in the case of configurations having a great variety of different pairs of final angular momenta, the orders of matrices for particular pairs of final angular momenta are several times smaller than they were for concrete $J$ while using the full basis of configurations. The more complex spectrum of the adjusted configuration is, the more efficient is this method.

Still one more approximation used in the calculations should be mentioned: all the configurations of a given ion, even those of different parity, are calculated in the same basis of ROs. If such an approach were used in the single-configuration approximation or at a small number of admixed configurations, it could introduce considerable bias into the energy spectrum and, correspondingly, into the eigenfunctions. While accounting for hundreds of admixed configurations the biases practically totally compensate. Meanwhile, the use of a single basis for the initial and final configurations allows one to avoid the biases related to the nonorthogonality of the basis used that are difficult to control and to compensate.

The methods briefly described in this section are quite efficient and reliable, which is confirmed by the multiple actual calculations of spectral characteristics. So, with the above-mentioned personal computer having $0.5 \mathrm{~GB}$ of RAM, the calculations were performed that would require a traditional basis of 400-500 thousand CSFs. As mentioned above, the methods applied nowadays allow one to use the restricted bases of CSFs, where the lower triangle of the matrix of nonrelativistic energy operator for a particular pair of $L S$ occupies practically the whole RAM of the computer. This fact gives the reasons to assume that the potential of further development of the programs in this direction is exhausted.

Meanwhile, there exists a method of an approximate account for the correlation effects in the energy spectra of atoms that does not lead to increase in the order of the matrices. It is based on employing the second order of perturbation theory and stems from papers $[57,58]$ that are devoted to explaining the nature of the semiempirical corrections. The matter lies in the fact that the total correction for the energy of a particular term may be written in the following form:

$$
\begin{aligned}
\Delta & E\left(K_{0} T_{0} L S, K\right) \\
= & \frac{\sum_{T}(2 L+1)(2 S+1) E^{2}\left(K_{0} T_{0} L S, K T L S\right)}{\bar{E}\left(K_{0}\right)-\bar{E}(K)} .
\end{aligned}
$$

The sum of squares of ICMEs in the numerator can be represented as the angular coefficients $f_{k}$ and $g_{k}$ together with the radial parts containing the products of integrals $R^{k}$. The angular coefficients that appear 
correspond to the angular coefficients of the matrix elements of an electrostatic interaction operator, except for the disappearance of the requirement of parity of a triad $\overline{l, k, l^{\prime}}$. It is the proportionality $f_{1} \sim L(L+1)$ that leads to the appearance of the most popular semiempirical correction. Such a property of the sum of squares of ICMEs allows one to take into account the correlation effects via the correlation corrections to the $F^{k}$, $G^{k}, R^{k}$ integrals and quantities similar to these. We have developed such an approach in a whole series of papers $[13,23,24,31,39,59]$. All the necessary analytical expressions can be found there. In doing this, the difference of the mean configuration energies may be defined in the conventional way (3.15) as well as with the account for the presence of ICMEs (3.16). The main advantage of the method described here is the low order of the calculated and diagonalized matrices, which is the reason for its wide application with the computers having small amount of RAM. Low orders of matrices, on their turn, lead to the main advantage of the method: similarly as with the semiempirical adjustment, the eigenfunctions do not contain the expansion in the whole basis of configurations and remain insufficiently accurate.

The described approximation has proved to be quite useful at performing the common configuration superposition, too. The complex of programs [53], the parts of which are used in our calculations, allows the existence of only up to five active shells. Meanwhile, the cases are known when the important admixed configurations have greater numbers of active shells. In such a case they may be taken into account by using the approximation described above.

\section{Examples of calculating the spectral characteristics of atoms and ions}

In the course of the seventies and the eighties a complex of our own original programs meant for calculating all spectral characteristics of atoms and ions was created at the Department of Theory of Atom. This included the solving of HF and HFJ equations as well as calculating the energy spectra and all the characteristics of electron transitions in the configuration superposition approximation. The programs for solving the Dirac-Hartree-Fock equations and the corresponding programs for calculating the spectral characteristics in the relativistic approximation were made as well. But the electronic computers used then had low power and a specific architecture, which determined the insufficient adaptability of the made FORTRAN programs to their immediate use on other computers. During the last decade, since the beginning of the nineties when the transition to PC began, the possibilities of PCs have grown tremendously. This has allowed using the programs from complex [55] that are widely known among the specialists. Nowadays while calculating the spectral characteristics of atoms, along with own programs written during the last years, usually the programs of solving the HF equations [60], the programs of calculating the angular parts of matrix elements of the energy $[61,62]$ and the transition [63] operators, and also the programs of calculating the transition characteristics [64] are used. Meanwhile, the programs [61, 62, 64] have been modified aiming both at the extension of their possibilities and at the different output of their results for using these in our programs. From our own programs, apart from the cited above [52,55], the most important of those not published yet are: the program for obtaining the transformed radial orbitals, the program for calculating the correlation corrections to the Slater integrals according to the second order of perturbation theory, the program for forming the energy operator matrix and diagonalizing it.

Let us treat the energy spectrum and the radiative lifetimes of the configuration $2 p^{4} 3 p$ of the $\mathrm{K} \mathrm{XI}$ ion from [49], presented in Table 3 as the first example. While calculating the energy spectrum the ROs of the single-electron states with $n \leq 3$ have been calculated in the Hartree-Fock approximation, and ROs with $4 \leq$ $n \leq 10$ have been obtained with the aid of the exponential transform (3.11). The selection of admixed configurations and switching to the refined basis has been performed as described in the previous section. The eigenfunctions and eigenvalues obtained have been used for calculating the characteristics of electric dipole transitions into all the lower-lying configurations. Two forms of the transition operator have been used: the "length" form (L) and the "velocity" form (V). While performing a wide $\mathrm{SC}$ the coincidence of the results obtained in using two forms of the operator may serve as a criterion of reliability of the obtained results. The radiative lifetimes have been obtained by summing up all the probabilities. As seen from Table 3, the values of SC energies taken from the theoretical value of the lowest level of the considered ion pretty well agree with the possessed experimental data [65]. There, a quite stable bias of $8000 \mathrm{~cm}^{-1}$ (i. e. about $0.28 \%$ of the total energy) is noted, which is characteristic of all $2 p^{4} n l$ configurations of this ion. After taking this bias into account, the theoretical values of energies coincide with all the possessed experimental data for this configura- 
Table 3. Energy levels and radiative lifetimes of $2 s^{2} 2 p^{4} 3 p$ K XI.

\begin{tabular}{|c|c|c|c|c|c|c|c|}
\hline \multirow[t]{2}{*}{ Level } & \multicolumn{2}{|c|}{ Energy $\left(\mathrm{cm}^{-1}\right)$} & \multirow[t]{2}{*}{ Composition (\%) } & \multicolumn{2}{|c|}{$\tau_{0}(\mathrm{~ns})$} & \multicolumn{2}{|c|}{$\tau_{t}(\mathrm{~ns})$} \\
\hline & Exp. [65] & $\mathrm{SC}$ & & $\mathrm{L}$ & $\mathrm{V}$ & $\mathrm{L}$ & $\mathrm{V}$ \\
\hline \multicolumn{8}{|l|}{$2 p^{4}\left({ }^{3} \mathrm{P}\right) 3 p$} \\
\hline${ }^{4} \mathrm{P}_{5 / 2}$ & 2785204 & 2794000 & 89 & 0.602 & 0.572 & 0.602 & 0.572 \\
\hline${ }^{4} \mathrm{P}_{3 / 2}$ & & 2796000 & 84 & 0.615 & 0.568 & 0.601 & 0.561 \\
\hline${ }^{4} \mathrm{P}_{1 / 2}$ & & 2804000 & 88 & 0.639 & 0.597 & 0.626 & 0.588 \\
\hline${ }^{4} \mathrm{D}_{7 / 2}$ & 2803297 & 2811000 & 97 & 0.399 & 0.416 & 0.399 & 0.416 \\
\hline${ }^{4} \mathrm{D}_{5 / 2}$ & 2808161 & 2816000 & $53+39\left({ }^{3} \mathrm{P}\right)^{2} \mathrm{D}$ & 0.464 & 0.472 & 0.464 & 0.472 \\
\hline${ }^{4} \mathrm{D}_{3 / 2}$ & & 2826000 & 85 & 0.427 & 0.429 & 0.427 & 0.426 \\
\hline${ }^{2} \mathrm{P}_{1 / 2}$ & & 2828000 & $\left.21+48\left({ }^{3} \mathrm{P}\right){ }^{4} \mathrm{D}+14\left({ }^{1} \mathrm{D}\right)\right)^{2} \mathrm{P}+10\left({ }^{3} \mathrm{P}\right)^{2} \mathrm{~S}$ & 0.537 & 0.529 & 0.504 & 0.507 \\
\hline${ }^{4} \mathrm{D}_{1 / 2}$ & & 2828846 & $48+16\left({ }^{3} \mathrm{P}\right){ }^{2} \mathrm{P}+13\left({ }^{3} \mathrm{P}\right){ }^{2} \mathrm{~S}+13\left({ }^{1} \mathrm{D}\right)^{2} \mathrm{P}$ & 0.479 & 0.489 & 0.415 & 0.414 \\
\hline${ }^{2} \mathrm{D}_{5 / 2}$ & 2824955 & 2833000 & $56+37\left({ }^{3} \mathrm{P}\right){ }^{4} \mathrm{D}$ & 0.456 & 0.486 & 0.456 & 0.486 \\
\hline${ }^{2} \mathrm{P}_{3 / 2}$ & & 2837000 & $41+24\left({ }^{3} \mathrm{P}\right)^{2} \mathrm{D}+22\left({ }^{1} \mathrm{D}\right)^{2} \mathrm{P}$ & 0.570 & 0.595 & 0.505 & 0.529 \\
\hline${ }^{4} \mathrm{~S}_{3 / 2}$ & & 2844000 & $62+18\left({ }^{3} \mathrm{P}\right)^{2} \mathrm{D}$ & 0.321 & 0.338 & 0.283 & 0.302 \\
\hline${ }^{2} \mathrm{D}_{3 / 2}$ & & 2847000 & $47+30\left({ }^{3} \mathrm{P}\right){ }^{4} \mathrm{~S}+11\left({ }^{3} \mathrm{P}\right)^{2} \mathrm{P}$ & 0.390 & 0.419 & 0.370 & 0.393 \\
\hline${ }^{2} \mathrm{~S}_{1 / 2}$ & & 2848000 & $72+15\left({ }^{3} \mathrm{P}\right)^{2} \mathrm{P}$ & 0.482 & 0.518 & 0.471 & 0.510 \\
\hline \multicolumn{8}{|l|}{$2 p^{4}\left({ }^{1} \mathrm{D}\right) 3 p$} \\
\hline${ }^{2} \mathrm{~F}_{5 / 2}$ & 2877507 & 2886000 & 65 & 0.515 & 0.510 & 0.515 & 0.510 \\
\hline${ }^{2} \mathrm{~F}_{7 / 2}$ & 2881197 & 2889000 & 97 & 0.475 & 0.497 & 0.475 & 0.497 \\
\hline${ }^{2} \mathrm{D}_{3 / 2}$ & 2897361 & 2905000 & 96 & 0.341 & 0.360 & 0.324 & 0.348 \\
\hline${ }^{2} \mathrm{D}_{5 / 2}$ & 2899633 & 2908000 & 95 & 0.333 & 0.356 & 0.333 & 0.356 \\
\hline${ }^{2} \mathrm{P}_{3 / 2}$ & & 2945000 & $58+38\left({ }^{3} \mathrm{P}\right)^{2} \mathrm{P}$ & 0.119 & 0.144 & 0.067 & 0.074 \\
\hline${ }^{2} \mathrm{P}_{1 / 2}$ & & 2952000 & $54+27\left({ }^{3} \mathrm{P}\right){ }^{2} \mathrm{P}+16\left({ }^{1} \mathrm{~S}\right)^{2} \mathrm{P}$ & 0.144 & 0.170 & 0.057 & 0.064 \\
\hline \multicolumn{8}{|l|}{$2 p^{4}\left({ }^{1} \mathrm{~S}\right) 3 p$} \\
\hline${ }^{2} \mathrm{P}_{3 / 2}$ & & 2984000 & 93 & 0.327 & 0.363 & 0.147 & 0.158 \\
\hline${ }^{2} \mathrm{P}_{1 / 2}$ & & 2988000 & $75+15\left({ }^{3} \mathrm{P}\right)^{2} \mathrm{P}$ & 0.209 & 0.244 & 0.175 & 0.206 \\
\hline
\end{tabular}

Table 4. Energy levels and radiative lifetimes of the twelve exited Ca IX.

\begin{tabular}{|c|c|c|c|c|c|c|c|c|c|}
\hline \multirow[t]{2}{*}{ Level } & \multicolumn{3}{|c|}{ Energy $\left(\mathrm{cm}^{-1}\right)$} & \multirow{2}{*}{$\begin{array}{c}\text { Composition } \\
(\%)\end{array}$} & \multicolumn{5}{|c|}{$\tau(\mathrm{ns})$} \\
\hline & Exp. [67] & SE [68] & $\mathrm{SC}$ & & $\mathrm{L}$ & $\mathrm{V}$ & Exp. [69] & MOPM [70] & MBPT [71] \\
\hline \multirow[t]{5}{*}{$3 p^{2}$} & 336245 & 336762 & 335800 & $76+19(3 s 3 d)^{1} \mathrm{D}$ & $8.72 \mathrm{E}-01$ & $9.07 \mathrm{E}-01$ & $0.840 \pm 0.060$ & & 1.06 \\
\hline & 339963 & 339754 & 339500 & 99 & $1.12 \mathrm{E}-01$ & $1.15 \mathrm{E}-01$ & & & 0.0636 \\
\hline & 341872 & 341740 & 341200 & 99 & $1.10 \mathrm{E}-01$ & $1.14 \mathrm{E}-01$ & & & 0.11 \\
\hline & 345472 & 345519 & 344500 & 94 & $1.12 \mathrm{E}-01$ & $1.18 \mathrm{E}-01$ & $0.134 \pm 0.008$ & & 0.107 \\
\hline & 398900 & 398776 & 398300 & 94 & $1.10 \mathrm{E}-01$ & $1.13 \mathrm{E}-01$ & $0.150 \pm 0.008$ & & 0.122 \\
\hline $3 s 3 d^{3} \mathrm{D}_{1}$ & 412078 & 412341 & 412400 & 99 & $7.55 \mathrm{E}-02$ & $7.79 \mathrm{E}-02$ & & & 0.0774 \\
\hline${ }^{3} \mathrm{D}_{2}$ & 412191 & 412582 & 412600 & 99 & $7.65 \mathrm{E}-02$ & $7.83 \mathrm{E}-02$ & $0.100 \pm 0.015$ & & 0.0787 \\
\hline${ }^{3} \mathrm{D}_{3}$ & 412405 & 412946 & 412900 & 99 & $7.81 \mathrm{E}-02$ & $7.90 \mathrm{E}-02$ & $0.117 \pm 0.009$ & & 0.0806 \\
\hline${ }^{1} \mathrm{D}_{2}$ & 467631 & 468131 & 468300 & $78+20\left(3 p^{2}\right)^{1} \mathrm{D}$ & $4.30 \mathrm{E}-02$ & $4.44 \mathrm{E}-02$ & $0.072 \pm 0.003$ & & 0.0454 \\
\hline $3 p 3 d^{3} \mathrm{~F}_{2}$ & 563714 & 563743 & 563900 & 96 & $4.28 \mathrm{E}-01$ & $4.28 \mathrm{E}-01$ & $0.653 \pm 0.063$ & & 0.431 \\
\hline${ }^{3} \mathrm{~F}_{3}$ & 565724 & 565974 & 565800 & 99 & $4.66 \mathrm{E}-01$ & $4.78 \mathrm{E}-01$ & $0.473 \pm 0.021$ & & 0.476 \\
\hline${ }^{3} \mathrm{~F}_{4}$ & 568194 & 568589 & 568100 & 99 & $4.52 \mathrm{E}-01$ & $4.79 \mathrm{E}-01$ & $0.513 \pm 0.040$ & & 0.459 \\
\hline${ }^{1} \mathrm{D}_{2}$ & 571900 & 571469 & 571800 & 96 & $9.93 \mathrm{E}-02$ & $1.02 \mathrm{E}-01$ & & & 0.0978 \\
\hline${ }^{3} \mathrm{P}_{2}$ & & 597263 & 598500 & $80+18^{3} \mathrm{D}$ & $6.08 \mathrm{E}-02$ & $6.24 \mathrm{E}-02$ & & & 0.0611 \\
\hline${ }^{3} \mathrm{P}_{1}$ & & 598115 & 599400 & $63+36{ }^{3} \mathrm{D}$ & $5.51 \mathrm{E}-02$ & $5.70 \mathrm{E}-02$ & & & 0.0548 \\
\hline${ }^{3} \mathrm{P}_{0}$ & & 599971 & 601000 & 99 & $6.34 \mathrm{E}-02$ & $6.68 \mathrm{E}-02$ & & & 0.0639 \\
\hline${ }^{3} \mathrm{D}_{1}$ & & 600981 & 601900 & $63+36{ }^{3} \mathrm{P}$ & $4.90 \mathrm{E}-02$ & $5.09 \mathrm{E}-02$ & & & 0.0506 \\
\hline${ }^{3} \mathrm{D}_{2}$ & 601204 & 601496 & 602500 & $81+18^{3} \mathrm{P}$ & $4.64 \mathrm{E}-02$ & $4.79 \mathrm{E}-02$ & & & 0.0474 \\
\hline${ }^{3} \mathrm{D}_{3}$ & 602704 & 601684 & 602700 & 99 & $4.42 \mathrm{E}-02$ & $4.52 \mathrm{E}-02$ & & & 0.0451 \\
\hline${ }^{1} \mathrm{~F}_{3}$ & & 650486 & 645400 & 98 & $4.05 \mathrm{E}-02$ & $4.13 \mathrm{E}-02$ & & & 0.0455 \\
\hline${ }^{1} \mathrm{P}_{1}$ & 618520 & 656335 & 655000 & 96 & $4.77 \mathrm{E}-02$ & $5.01 \mathrm{E}-02$ & & & 0.0524 \\
\hline \multirow{2}{*}{$3 s 4 s{ }^{3} \mathrm{~S}_{1}$} & 760838 & & 761800 & 99 & $1.50 \mathrm{E}-02$ & $1.51 \mathrm{E}-02$ & & 0.0150 & \\
\hline & 774480 & & 775800 & 97 & $2.39 \mathrm{E}-02$ & $2.39 \mathrm{E}-02$ & & 0.0244 & \\
\hline
\end{tabular}


Table 4. (Continued.)

\begin{tabular}{|c|c|c|c|c|c|c|c|c|}
\hline \multirow[t]{2}{*}{ Level } & \multicolumn{2}{|c|}{ Energy $\left(\mathrm{cm}^{-1}\right)$} & \multirow{2}{*}{$\begin{array}{c}\text { Composition } \\
(\%)\end{array}$} & \multicolumn{5}{|c|}{$\tau(\mathrm{ns})$} \\
\hline & Exp. [67] SE [68] & $\mathrm{SC}$ & & $\mathrm{L}$ & $\mathrm{V}$ & Exp. [69] & MOPM [70] & MBPT [71] \\
\hline $3 s 4 p^{3} \mathrm{P}_{0}$ & & 831600 & 98 & $7.23 \mathrm{E}-02$ & $7.35 \mathrm{E}-02$ & & 0.0721 & \\
\hline${ }^{3} \mathrm{P}_{1}$ & & 831800 & $86+12{ }^{1} \mathrm{P}$ & $5.14 \mathrm{E}-02$ & $5.26 \mathrm{E}-02$ & & 0.0517 & \\
\hline $3 d^{2} \quad{ }^{3} \mathrm{~F}_{2}$ & & 832000 & 99 & $4.76 \mathrm{E}-02$ & $4.88 \mathrm{E}-02$ & & & 0.0373 \\
\hline${ }^{3} \mathrm{~F}_{3}$ & & 832300 & 99 & $4.82 \mathrm{E}-02$ & $4.90 \mathrm{E}-02$ & & & 0.0491 \\
\hline${ }^{3} \mathrm{~F}_{4}$ & & 832600 & 99 & $4.91 \mathrm{E}-02$ & $4.93 \mathrm{E}-02$ & & & 0.0500 \\
\hline $3 s 4 p^{3} \mathrm{P}_{2}$ & & 833100 & 99 & $7.25 \mathrm{E}-02$ & $7.24 \mathrm{E}-02$ & & 0.0726 & \\
\hline${ }^{1} \mathrm{P}_{1}$ & 832314 & 833900 & $83+12{ }^{3} \mathrm{P}$ & $1.90 \mathrm{E}-02$ & $1.92 \mathrm{E}-02$ & & 0.0200 & \\
\hline $3 d^{2}{ }^{1} \mathrm{D}_{2}$ & & 850600 & 97 & $3.96 \mathrm{E}-02$ & $4.14 \mathrm{E}-02$ & & & 0.0375 \\
\hline${ }^{1} \mathrm{G}_{4}$ & & 850800 & 98 & $9.17 \mathrm{E}-02$ & $8.73 E-02$ & & & 0.102 \\
\hline${ }^{3} \mathrm{P}_{0}$ & & 853700 & 99 & $3.69 \mathrm{E}-02$ & $3.90 \mathrm{E}-02$ & & & 0.0362 \\
\hline${ }^{3} \mathrm{P}_{1}$ & & 853800 & 99 & $3.70 \mathrm{E}-02$ & $3.90 \mathrm{E}-02$ & & & 0.0434 \\
\hline${ }^{3} \mathrm{P}_{2}$ & & 854000 & 98 & $3.74 \mathrm{E}-02$ & $3.92 \mathrm{E}-02$ & & & 0.0324 \\
\hline${ }^{1} \mathrm{~S}_{0}$ & & 902300 & 95 & $3.22 \mathrm{E}-02$ & $3.48 \mathrm{E}-02$ & & & 0.0073 \\
\hline $3 s 4 d^{3} \mathrm{D}_{1}$ & 917200 & 918700 & 96 & $2.06 \mathrm{E}-02$ & $2.07 \mathrm{E}-02$ & & & \\
\hline${ }^{3} \mathrm{D}_{2}$ & 917314 & 918800 & 96 & $2.06 \mathrm{E}-02$ & $2.08 \mathrm{E}-02$ & & & \\
\hline${ }^{3} \mathrm{D}_{3}$ & 917528 & 919000 & 96 & $2.05 \mathrm{E}-02$ & $2.10 \mathrm{E}-02$ & & & \\
\hline${ }^{1} \mathrm{D}_{2}$ & 921921 & 920300 & 95 & $4.00 \mathrm{E}-02$ & $4.10 \mathrm{E}-02$ & & & \\
\hline $3 p 4 s{ }^{3} \mathrm{P}_{0}$ & 941471 & 943000 & 99 & $2.23 \mathrm{E}-02$ & $2.27 \mathrm{E}-02$ & & & \\
\hline${ }^{3} \mathrm{P}_{1}$ & 942658 & 944100 & 96 & $2.20 \mathrm{E}-02$ & $2.23 \mathrm{E}-02$ & & & \\
\hline${ }^{3} \mathrm{P}_{2}$ & 946378 & 947300 & 99 & $2.24 \mathrm{E}-02$ & $2.25 \mathrm{E}-02$ & & & \\
\hline${ }^{1} \mathrm{P}_{1}$ & & 956400 & 91 & $1.45 \mathrm{E}-02$ & $1.45 \mathrm{E}-02$ & & & \\
\hline $3 s 4 f^{3} \mathrm{~F}_{2}$ & 954594 & 956700 & 99 & $8.24 \mathrm{E}-03$ & $8.33 \mathrm{E}-03$ & & & \\
\hline${ }^{3} \mathrm{~F}_{3}$ & 954594 & 956800 & 99 & $8.24 \mathrm{E}-03$ & $8.33 \mathrm{E}-03$ & & & \\
\hline${ }^{3} \mathrm{~F}_{4}$ & 954594 & 956800 & 99 & $8.24 \mathrm{E}-03$ & $8.33 \mathrm{E}-03$ & & & \\
\hline${ }^{1} \mathrm{~F}_{3}$ & 963050 & 965600 & 98 & $8.72 \mathrm{E}-03$ & $8.82 \mathrm{E}-03$ & & & \\
\hline $3 p 4 p{ }^{1} \mathrm{P}_{1}$ & & 999500 & 90 & $2.31 \mathrm{E}-02$ & $2.40 \mathrm{E}-02$ & & & \\
\hline${ }^{3} \mathrm{D}_{1}$ & & 1005600 & 86 & $2.76 \mathrm{E}-02$ & $2.87 \mathrm{E}-02$ & & & \\
\hline${ }^{3} \mathrm{D}_{2}$ & 1005234 & 1006800 & 94 & $2.81 \mathrm{E}-02$ & $2.91 \mathrm{E}-02$ & & & \\
\hline${ }^{3} \mathrm{D}_{3}$ & 1008574 & 1009800 & 96 & $2.78 \mathrm{E}-02$ & $2.89 \mathrm{E}-02$ & & & \\
\hline${ }^{3} \mathrm{P}_{0}$ & 1010894 & 1012000 & 99 & $2.70 \mathrm{E}-02$ & $2.79 \mathrm{E}-02$ & & & \\
\hline${ }^{3} \mathrm{P}_{1}$ & 1012034 & 1013000 & 91 & $2.70 \mathrm{E}-02$ & $2.78 \mathrm{E}-02$ & & & \\
\hline${ }^{3} \mathrm{P}_{2}$ & 1014384 & 1015100 & 97 & $2.71 \mathrm{E}-02$ & $2.77 \mathrm{E}-02$ & & & \\
\hline${ }^{3} \mathrm{~S}_{1}$ & 1015624 & 1017000 & 91 & $2.58 \mathrm{E}-02$ & $2.76 \mathrm{E}-02$ & & & \\
\hline${ }^{1} \mathrm{D}_{2}$ & & 1026700 & 93 & $2.48 \mathrm{E}-02$ & $2.49 \mathrm{E}-02$ & & & \\
\hline${ }^{1} \mathrm{~S}_{0}$ & & 1045000 & 94 & $3.15 \mathrm{E}-02$ & $3.19 \mathrm{E}-02$ & & & \\
\hline
\end{tabular}

tion with the accuracy of four significant numbers. This allows one to assume that the energies of the levels that are presently unknown are foretold with a high accuracy. From the data on the composition of eigenfunctions given in the table it is seen that part of levels corresponds well to $L S$-coupling, while some levels have such high mixing that their classical notation (the first column) is at a complete variance with the maximum weight.

The complications in the experimental determination of the levels of configurations $2 s^{2} 2 p^{N-1} 3 p$ may be in part related to the fact that the two-electron transitions from some of their levels to the levels of configurations $2 s 2 p^{N+1}$ have quite high probabilities, whereas in experiment these transitions usually are not registered. The importance of two-electron transitions is well understood from the data on the lifetimes from Table 3. The lifetimes that are obtained by summing up all the transitions $\left(\tau_{t}\right)$ and the ones obtained without the account of two-electron transitions $\left(\tau_{0}\right)$ are given there. As seen from the table, the account of two-electron transitions sometimes lessens the lifetimes more than twice. It is characteristic that all up to now experimentally determined levels does not possess any notable transitions to the level ${ }^{2} \mathrm{~S}$ of the configuration $2 s 2 p^{6}$. The comparison of lifetimes obtained while using two forms of the transition operator ( $\mathrm{L}$ and $\mathrm{V}$ ) shows their good agreement and indicates that the per- 
formed superposition of configurations in the basis of TROVP has allowed obtaining not just the energy values with high accuracy, but fairly reliable eigenfunctions as well.

The energy spectrum and the radiative lifetimes of 12 excited configurations of the Ca IX ion are presented in Table 4 as the second example. The presented data were obtained in the same approximation as in the calculation above. Still, one peculiarity exists. For a long time, there was no success in obtaining good agreement of the theoretical energy levels with the experimental ones for the configurations containing the electrons with Ca IX. In the case of these configurations the deviation from the experimental data on energies has been sharp. The calculations indicate that it is necessary to take into account the virtual excitations from the $2 s^{2}$-shell. However, then the admixed configurations with 6 active shells arise, e.g., $2 s^{2} 2 p^{4} 3 s 3 p n l n^{\prime} l^{\prime}$ or $2 s 2 p^{5} 3 s 3 p n l n^{\prime} l^{\prime}$. Meanwhile, the programs from the complex [55] that we use for calculating the angular parts of the matrix elements allow for no more than 5 active shells. Therefore, the energy corrections due to the configurations with 6 active shells have been taken into account in a simplified manner: in the second order of perturbation theory [40]. Besides the theoretical (SC) results, this table also gives the experimental data on the experimental levels taken from the NIST Atomic Spectra Database [67] and the semiempirical results (SE) from [68] obtained by the optimization of theoretically calculated Slater parameters for consistency with the established energy levels. The table also contains the weights (those exceeding 10\%) of the most important terms composing the given level. The analysis of the weight coefficients demonstrates that for most levels the $L S$-coupling is a quite acceptable approximation. Two levels ${ }^{1} \mathrm{D}_{2}$ from configurations $3 p^{2}$ and $3 s 3 d$ should be especially noted. The very strong mixing of these levels is determined by the fact that both configurations are odd and located quite closely in the energy spectra. Such mixing has a large influence upon the characteristics of the transitions related with these levels. As seen from Table 4, the theoretical energy levels agree rather well with the available experimental data [31] and with the semiempirical results [10]. Only the energy level $3 p 3 d^{1} \mathrm{P}_{1}$ differs from the experimental value significantly, the relative difference is about $0.2 \%$. The experimental energy of this level agrees well with the semiempirical one.

Table 4, like the previous one, contains the radiative lifetimes obtained while using the two forms of the transition operator. There they demonstrate quite good agreement. This table also contains the available experimental data [69] and the theoretical results of the method of the optimized potential model (MOPM) [70], as well as the many body perturbation theory (MBPT) [71]. As mentioned before for the lifetimes of configuration $2 s^{2} 2 p^{N-1} 3 p$, in this case the twoelectron transitions are also very important for the lifetimes of levels from which these transitions may occur. If the two-electron transitions were not taken into account, the lifetimes of levels $3 s 4 p{ }^{1} \mathrm{P}_{1}, 3 p 4 s{ }^{1} \mathrm{P}_{1}$, and $3 s 4 f^{1} \mathrm{~F}_{3}$ would be about $20 \%$ larger. Our lifetimes are more or less larger than the experimental ones. The lifetimes obtained by the MBPT exceed the experimental values, too, but they coincide with our results for these levels quite well. The results of SC very well agree with the MOPM when available.

\section{Conclusion}

More than fifty years have passed since the elaboration of the multiconfiguration Hartree-Fock-Jucys equations, but their solutions or the transformed radial orbitals that closely approximate the above ones still are the most efficient and popular bases used while performing the superposition of configurations. During this time interval the possibilities both of the computers used and the programs applied for the atomic calculations have grown tremendously. While the first applications of the multiconfiguration approximation have been limited to the superposition of just a few and sometimes only two configurations, nowadays in the common calculations performed using just the simple PCs many hundreds and even thousands of admixed configurations are taken into account, and the number of CSFs occurring there in the traditional basis may reach a million. The accuracy of ab initio calculations achieved there allows one to easily rectify the biases of the experimental identification of energy levels, and in the characteristics of the electron transitions (oscillator strengths, radiative lifetimes) enables one to obtain the data even superior in accuracy than those of experiment. Still, the obtainable reliability is not a limit and the development of theory is under way. It seems plausible that using the solutions of the corresponding quasi-relativistic equations in the multiconfiguration approximation will be an essential step that will extend considerably the field of application of the method without its significant complication. 


\section{References}

[1] A. Jucys, Fock equations in a multiconfiguration approach, JETP 23, 2(8), 129-139 (1952) [in Russian].

[2] A. Bolotin and A. Jucys, Application of a multiconfiguration approach in definition of dipole strength for beryllium- and boron-like atoms, JETP 24(5), 537-544 (1953) [in Russian].

[3] V. Kaveckis and A. Jucys, Analytic single-electron wave functions in a multiconfiguration approach for beryllium- and boron-like atoms, JETP 25(3(9)), 257263 (1953) [in Russian].

[4] V. Kybartas and A. Jucys, Solution of Fock equations for beryllium atom in two-configuration approach, JETP 25(3(9)), 264-270 (1953) [in Russian].

[5] G. Ciunaitis and A. Jucys, Two-configuration approach for $1 s^{2} 2 s^{2} 2 p 3 s$ configuration of carbon atom, JETP 25(6(12)), 679-682 (1953) [in Russian].

[6] A. Jucys, V. Kybartas, and I. Glembockis, Solution of simplified Fock equations in two-configuration approach for beryllium-like atoms, JETP 27(4(10)), 425430 (1954) [in Russian].

[7] V. Kybartas, V. Kaveckis, and A. Jucys, Self-consistent Fock field in three-configuration approach for boron atom, JETP 29(5(11)), 623-628 (1955) [in Russian].

[8] R. Karazija, Numerical solution of Hartree-Fock equations, in: Scientific Conference of Young Scientists (Vilnius, 1967) pp. 87-89 [in Russian].

[9] R. Karazija, P. Bogdanovich, and A. Jucys, On the numerical solution of Hartree-Fock equations independent of coupling sheme, Acta Phys. Hung. 27, 467-475 (1969).

[10] P. Bogdanovich, Program for numerical solution of Hartree-Fock equations, in: Collection of Programs for the Automation of Atomic Calculations, Vol. 2 (Institute of Physics, Vilnius, 1977) 96 p. [in Russian].

[11] P. Bogdanovich and A. Jucys, Numerical solution of Hartree-Fock equations in single- and two-configuration approach, in: All-Union Seminar on Theory of Atoms and Atomic Spectra, Abstracts, Liet. Fiz. Rink. 12(4), 697 (1972) [in Russian].

[12] P. Bogdanovich and A. Jucys, Self-consistent HartreeFock field in tetra-configuration approach for $\mathrm{Pr}^{3+}$, in: Proceedings of All-Union Seminar on Theory of Atoms and Atomic Spectra (Moscow, 1972) pp. 64-68 [in Russian].

[13] P.O. Bogdanovich and G.L. Zukauskas, Approximate allowance for superposition of configurations in atomic spectra, Sov. Phys. - Collection 23(3), 13-25 (1983).

[14] V. Fock, Näherungsmethods zur Lösung des quantenmechanischen Mehrkörperproblems, Z. Phys. B 61, 126-148 (1930).

[15] Ch. Froese-Fischer, The Hartree-Fock Method for Atoms (Wiley, New York, 1977) 308 p.

[16] C.F. Fischer, T. Brage, and P. Jönsson, Computational Atomic Structure. An MCHF Approach (Institute of
Physics Publishing, Bristol and Philadelphia, 1997) $280 \mathrm{p}$.

[17] A. Jucys, J. Vizbaraite, T. Strockyte, and A. Bandzaitis, On multiconfiguration approach and its convergence, Opt. Spektrosk. (USSR) 12(2), 157-162 (1962) [in Russian].

[18] P. Bogdanovich, On the problem of usage of transformed functions for approach of solutions of multiconfiguration Hartree-Fock-Jucys equations, in: Processes in Inner Shells of Atoms (Moscow, Scientific Council on Spectroscopy, 1986) pp. 90-111 [in Russian].

[19] P. Bogdanovich and J. Boruta, On the numerical solution of the Hartree-Fock-Jucys equations, Sov. Phys. - Collection 16(1), 34-70 (1976).

[20] P.O. Bogdanovicius, J.I. Boruta, and J.I. Vizbaraite, Calculation of the probabilities of $n p^{5} n^{\prime} p \rightarrow n p^{5} n^{\prime} s$ dipole transitions for the $\mathrm{Ne}$ I and Ar I spectra in the two-configuration approach, Sov. Phys. - Collection 16(2), 42-45 (1976).

[21] A. Jucys, J . Vizbaraite, J. Batarunas, and V. Kaveckis, On multiconfiguration approach and its further development, Proceedings of Lithuanian Academy of Sciences B 2(14), 3-16 (1958) [in Russian].

[22] M. Bogdanovichiene and P. Bogdanovich, Solution of Hartree-Fock-Jucys equations for highly charged ions, in: 6th All-Union Conference on Theory of Atom, Abstracts (Voronezh, 1980) [in Russian].

[23] P.O. Bogdanovich, G.L. Zukauskas, and S.D. Sadziuviene, Role of atomic energy correlation corrections defined by configurations of the type $n_{1} l_{1}^{N_{1}-1} n_{2} l_{2}^{N_{2}} n_{3} l_{3}$ and $n_{3} l_{3}^{4 l_{3}+1} n_{1} l_{1}^{N_{1}+1} n_{2} l_{2}^{N_{2}}$, Sov. Phys. - Collection 24(5), 20-27 (1984).

[24] P. Bogdanovich, G. Zhukauskas, and S. Sadziuviene, Energy spectra of rare earth elements ions accounting correlation corrections, in: Many-Particle Effects in Atoms (Moscow, Scientific Council on Spectroscopy, 1985) pp. 39-86 [in Russian].

[25] P. Bogdanovich, R. Lukoshiavichius, A. Nikitin, Z. Rudzikas, and A. Holtygin, Lines of carbon, nitrogen and oxygen ions in planetary nebulae spectra, I. Transition probabilities and oscillator strengths, Astrophysics 22(3), 551-562 (1985) [in Russian].

[26] P.O. Bogdanovich, G.L. Zukauskas, A.P. Momkauskaite, and V.I. Tutlys, Calculation of radiation lifetimes of Ar II and Kr II energy levels, Sov. Phys. - Collection 25(6), 33-40 (1985).

[27] K. Blagoev, P. Bogdanovich, N. Dimitrov, B. Amar, A. Momkauskaite, and Z. Rudzikas, Two-electron transitions between $n d^{8}(n+1) s^{2}-n d^{9}(n+1) p$ configurations in the spectra of $\mathrm{Cu}^{+}, \mathrm{Ag}^{+}, \mathrm{Au}^{+}, \mathrm{Zn}^{2+}, \mathrm{Cd}^{2+}$, $\mathrm{Hg}^{2+}$, Physica Scripta 41(2), 213-216 (1990).

[28] P. Bogdanovich, G. Tautvaišienė, Z. Rudzikas, and A. Momkauskaitè, A simple method of accounting for 
correlation effects in electron transitions and its application in finding oscillator strengths and the solar abundance of zirconium, Mon. Not. R. Astron. Soc. 280, 95-102 (1996).

[29] P. Bogdanovich, A.K. Kazansky, and V.N. Ostrovsky, Near-threshold double photoionization: $\mathrm{Kr}+\gamma \rightarrow$ $\mathrm{Kr}^{2+}\left({ }^{3} \mathrm{P}^{\mathrm{e}}\right)+\mathrm{e}+\mathrm{e}$, J. Phys. B 30, 921-940 (1997).

[30] M.-C. Buchet-Poulizac, P. Bogdanovich, and E.J. Knystautas, Spectroscopic study of doubly excited levels in Ne VII** and Ne VIII**, J. Phys. B 34, 233-243 (2001).

[31] P. Bogdanovich, G. Gaigalas, A. Momkauskaite, and Z. Rudzikas, Accounting for admixed configurations in the second order of perturbation theory for complex atoms, Physica Scripta 56, 230-239 (1997).

[32] P. Bogdanovich and P. Vaitiekunas, Calculation by dipole static polarizabilities of the light atoms and ions by the transformed functions, Sov. Phys. - Collection 25(3), 15-23 (1985).

[33] P.O. Bogdanovich, Usage of transformed functions for calculations of electric dipole transitions, Lithuanian Phys. J. 31(2), 79-83 (1991).

[34] P. Bogdanovich and G. Dushkesas, Correlation correction for the electron density at the nuclei of light atoms, Lithuanian Phys. J. 38(1), 122-126 (1998).

[35] P. Bogdanovich, S. Shadzhiuviene, and N. Piskunova, Investigation of properties of transformed radial orbitals, Lithuanian Phys. J. 38(2), 145-151 (1998).

[36] P. Bogdanovich and R. Karpushkiene, Transformed radial orbitals with a variable parameter for the configuration interaction, Lithuanian Phys. J. 39(3), 154-169 (1999).

[37] R. Karazija, Sums of Atomic Quantities and Mean Characteristics of Spectra (Mokslas, Vilnius, 1991) 272 p. [in Russian].

[38] S. Kucas, V. Jonauskas, and R. Karazija, Global characteristics of atomic spectra and their use for the analysis of spectra, IV. Configuration interaction effects, Physica Scripta 55, 667-675 (1997).

[39] R. Karpuškienè, R. Karazija, and P. Bogdanovich, The average energy distance between two interacting configurations and its application for the investigation of CI in atoms, Physica Scripta 64, 333-342 (2001).

[40] P. Bogdanovich, R. Karpuškienè, and Z. Rudzikas, Calculation of electronic transitions in S IX, Physica Scripta T80, 474-475 (1999).

[41] P. Bogdanovich, R. Karpushkiene, A. Momkauskaite, and A. Udris, Theoretical calculation of wavelengths and oscillator strengths of $2 p-3 l$ transitions in $\mathrm{Mg} \mathrm{V}$, Si VII, and S IX ions, Lithuanian J. Phys. 39(1), 9-23 (1999).

[42] P. Bogdanovich and I. Martinson, Theoretical transition probabilities for Au II, Physica Scripta 61, 142145 (2000).

[43] P. Bogdanovich, R. Karpuškienè, and A. Momkauskaite, Calculation of electron transitions in the $2 p^{3}$ con- figuration of Ar XII and Ca XIV, Lithuanian J. Phys. 40(4), 248-252 (2000).

[44] P. Bogdanovich, A. Kyniene, R. Karazija, R. Karpuškienè, and G. Gaigalas, Additional symmetry for the electronic shell in its ground state and manyelectron effects, Eur. Phys. J. D 11, 175-183 (2000).

[45] R. Karpuškienè and P. Bogdanovich, Oscillator strengths of Zn I and Zn II, Lithuanian J. Phys. 41(3), 174-176 (2001).

[46] P. Bogdanovich, R. Karpuškienè, and I. Martinson, $A b$ initio wavelengths and oscillator strengths for $\mathrm{Cl} \mathrm{X}$, Physica Scripta 67, 44-51 (2003).

[47] P. Bogdanovich, R. Karpuškienè, and I. Martinson, Theoretical lifetimes for all states of five $\mathrm{Cl} \mathrm{X}$ configurations, Nucl. Instrum. Methods B 205, 70-73 (2003).

[48] P. Bogdanovich, R. Karpuškienè, and A. Udris, Theoretical study of the energy spectrum of the $2 p^{3} 3 p$ in $\mathrm{S}$ IX and related electron transitions, Physica Scripta 67, 395-400 (2003).

[49] R. Karpuškienè and P. Bogdanovich, Two-electron transitions and their importance for lifetimes of levels of configuration $2 p^{4} 3 p$ in K XI, J. Phys. B 36, 21452152 (2003).

[50] R. Karpuškienè, P. Bogdanovich, and A. Udris, Ab initio oscillator strengths and transition probabilities of transitions from $2 s^{2} 2 p^{2} 3 l$ and $2 s 2 p^{3} 3 l$ in $\mathrm{S} \mathrm{X}$, At. Data Nucl. Data Tables (in press).

[51] P. Bogdanovich and R. Karpuškienè, Numerical methods of the preliminary evaluation of the role of admixed configurations in atomic calculations, Comput. Phys. Commun. 134, 321-334 (2001).

[52] P. Bogdanovich, R. Karpuškienè, and A. Momkauskaite, A program of generation and selection of configurations for the configuration interaction method in atomic calculations SELECTCONF, Comput. Phys. Commun. (submitted).

[53] Ch. Froese Fischer, The MCHF atomic-structure package, Comput. Phys. Commun. 128, 635-636 (2000).

[54] P. Bogdanovich, R. Karpuškienè, and A. Momkauskaite, Some problems of calculation of energy spectra of complex atomic configurations, Comput. Phys. Commun. 143, 174-180 (2002).

[55] P. Bogdanovich and A. Momkauskaite, A program for generating configuration state lists in many-electron atoms, Comput. Phys. Commun. (submitted).

[56] P. Bogdanovich, S. Shadzhiuviene, and V. Tutlys, Partial diagonalization of large energy matrices, Lithuanian J. Phys. 36(4), 312-314 (1996).

[57] K. Rajnak and B.G. Wybourne, Configuration interaction effects in $l^{N}$ configurations, Phys. Rev. 132, 280 (1963).

[58] B.G. Wybourne, Generalization of "Linear theory" of configuration interaction, Phys. Rev. 137 A364 (1965).

[59] P. Bogdanovich, G. Gaigalas, and A. Momkauskaite, Accounting for correlation corrections to interconfig- 
urational matrix elements, Lithuanian Phys. J. 38(5), 368-374 (1998).

[60] C.F. Fischer, A general Hartree-Fock program, Comput. Phys. Commun. 43, 355-363 (1987).

[61] A. Hibbert, R. Glass, and C.F. Fischer, A general program for computing angular integrals of the BreitPauli Hamiltonian, Comput. Phys. Commun. 64(3), 455-472 (1991).

[62] G. Gaigalas, The library of subroutines for calculation of matrix elements of two-particle operators for many-electron atoms, Lithuanian J. Phys. 42(2), 73-86 (2002).

[63] C.F. Fischer, M.R. Godefroid, and A. Hibbert, A program for performing angular integrations for transitions operators, Comput. Phys. Commun. 64(3), 486500 (1991).

[64] C.F. Fischer and M.R. Godefroid, Programs for computing $L S$ and $L S J$ transitions from MCHF wave functions, Comput. Phys. Commun. 64(3), 501-519 (1991).
[65] C. Jupén and E. Träbert, The $2 p^{4} 3 s$ and $3 d$ configurations in K XI, J. Phys. B 34, 3053-3061 (2001).

[66] P. Bogdanovich, R. Karpuškienè, and A. Udris, $A b$ initio oscillator strengths and radiative lifetimes for Ca IX, J. Phys. B. (submitted).

[67] http://www.physics.nist.gov/cgi-bin/ AtData/main_asd

[68] B.C. Fawcett, Calculated oscillator strengths and wavelengths for allowed transitions within the third shell for ions in the Mg-like isoelectronic sequence between S V and Ni XVII, At. Data Nucl. Data Tables 28, 579-596 (1983).

[69] E. Träbert et al., Beam-foil study of the lifetimes of $n=3$ levels in Na-like Ca X, Mg-like Ca IX and Silike Ca VII, J. Phys. B 29, 2647-2659 (1996).

[70] S.S. Tayal, Oscillator strengths for Ar VII, Ca IX and Fe XV, J. Phys. B 19, 3421-3430 (1986).

[71] U.I. Safronova, W.R. Johnson, and H.G. Berry, Excitation energies and transition rates in magnesiumlike ions, Phys. Rev. A 61, 052503-1-11 (2000).

\section{DAUGIAKONFIGŪRACINIO ARTUTINUMO TAIKYMAS ATOMINIAMS SPEKTRAMS TIRTI}

\section{P. Bogdanovich}

VU Teorines fizikos ir astronomijos institutas, Vilnius, Lietuva

\section{Santrauka}

Apžvelgta daugiakonfigūracinio artutinumo metodų, sukurtų Teorinès fizikos ir astronomijos instituto Atomo teorijos skyriuje atomų bei jonų spektrinèms charakteristikoms tirti, plètra per pastaruosius penkiasdešimt metų, pradedant nuo to laiko, kai prof. A. Jucys sukūrè daugiakonfigūracines lygtis. Trumpai aptartos tụ lygčiu sprendiniu savybės bei galimybė juos pakeisti transformuo- tomis radialiosiomis orbitalemis. Taip pat aprašyti būdai, leidžiantys efektyviai diagonalizuoti dideles energijos operatoriaus matricas, jeigu tik apatinis ju trikampis telpa kompiuterio operatyviojoje atmintyje, ir metodai, leidžiantys efektyviai parinkti pataisines konfigūracijas bei dešimtimis kartų mažinti naudojamu termu rinkinius. Aprašytų metodų taikymas iliustruotas keliais pavyzdžiais. 